\title{
AROUND TRACE FORMULAS IN NON-COMMUTATIVE INTEGRATION
}

\author{
SHIGERU YAMAGAMI \\ Graduate School of Mathematics \\ Nagoya University \\ Nagoya, 464-8602, JAPAN
}

\begin{abstract}
Trace formulas are investigated in non-commutative integration theory. The main result is to evaluate the standard trace of a Takesaki dual and, for this, we introduce the notion of interpolator and accompanied boundary objects. The formula is then applied to explore a variation of Haagerup's trace formula.
\end{abstract}

\section{INTRODUCTION}

The Haagerup's trace formula in non-commutative integration is a key to his whole theory of non-commutative $L^{p}$-spaces (see [6] and [9]). Our purpose here is to analyse it from the view point of modular algebras (10, [11), which was originally formulated in terms of Haagerup's $L^{p}$-theory itself. So, to circumvent tautological faults and also to fix notations, we first describe modular algebras as well as standard Hilbert spaces in terms of basic ingredients of Tomita-Takesaki theory.

The semifiniteness of Takesaki's duals is then established by constructing relevant Hilbert algebras as a collaboration of modular algebras and complex analysis. Note that the known proofs of the existence of standard traces are not direct; for example, it is usually deduced from the innerness of modular automorphism groups combined with a reverse Radon-Nikodym theorem such as Pedersen-Takesaki's or Connes'.

Since our construction of the Hilbert algebras is based on complex analysis, the associated trace can be also described in a calculational way. To make the setup transparent, we introduce the notion of interpolators together with associated boundary operators and vectors. Viewing things this way, the main trace formula turns out to be just a straightforward consequence of definitions. The Haagerup's trace formula is then derived in a somewhat generalized form as a concrete application of our formula.

The Haagerup's correspondence between normal functionals and relatively invariant measurable operators on Takesaki's duals is also established on our streamlines.

Recall that the standard approach to these problems is by the theory of operatorvalued weights ([4, [5]) coupled with dual weights ([2], 3]), which is based on extended positive parts, a notion of metaphysical flavor, and somewhat elaborate. Our method may not provide an easy route either but can be applied rather straightforwardly; it is just a simple combination of elementary Fourier calculus and complex analytic nature of modular stuffs. 
The presentation below originates from the author's old work in 1990, which was addressed on the occasion of a satellite meeting of ICM90 held at Niigata University. The author would like to express hearty gratitude to Kichisuke Saito for his organization of the meeting and these records.

Notation and Convention: The positive part of a $\mathrm{W}^{*}$-algebra $M$ (resp. its predual $\left.M_{*}\right)$ is denoted by $M_{+}\left(\operatorname{resp} . M_{*}^{+}\right)$.

For a positive element $p$ in $M_{+}$or $M_{*}^{+}$, its support projection in $M$ is denoted by $[p]$.

For a functional $\varphi \in M_{*}^{+}$, the associated GNS-vector in the standard Hilbert space $L^{2}(M)$ of $M$ is denoted by $\varphi^{1 / 2}$ (natural notation though not standard) and the modular operator by $\Delta_{\varphi}$ so that $\Delta_{\varphi}\left(a \varphi^{1 / 2}\right)=\varphi^{1 / 2} a$ for $a \in[\varphi] M[\varphi]$.

For $\varphi, \psi \in M_{*}^{+}, \sigma_{t}^{\varphi, \psi}$ stands for the relative modular group of $[\varphi] M[\psi]$, which is simply denoted by $\sigma_{t}^{\varphi}$ and expresses a modular automorphism group of the reduced algebra $[\varphi] M[\varphi]$ when $\varphi=\psi$.

For convergence in $M, \mathrm{w}^{*}$-topology (resp. s-topology or $\mathrm{s}^{*}$-topology) means weak operator topology (resp. strong operator topology or *strong operator topology) as a von Neumann algebra on the standard Hilbert space $L^{2}(M)$.

Direct integrals are indicated by $\oint$ instead of ordinary $\int^{\oplus}$. This is to avoid duplication of sum meanings.

The notion of weights is used in a very restrictive sense: weights are orthogonal sums of functionals in $M_{*}^{+}$.

For an interval $I$ contained in $[0,1], T_{I}$ expresses the tubular domain based on an imaginary trapezoid $\left\{(x, y) \in \mathbb{R}^{2} ; x \leq 0, y \leq 0,-(x+y) \in I\right\}: T_{I}=\{(z, w) \in$ $\left.\mathbb{C}^{2} ; \operatorname{Im} z \leq 0, \operatorname{Im} w \leq 0,-(\operatorname{Im} z+\operatorname{Im} w) \in I\right\}$.

A function $f: D \rightarrow M$ with $D \subset \mathbb{C}$ is said to be $\mathrm{w}^{*}$-analytic (s*-analytic) if it is $\mathrm{w}^{*}$-continuous ( $\mathrm{s}^{*}$-continuous) and holomorphic when restricted to the interior $D^{\circ}$. Note that topologies are irrelevant for holomorphicity because weaker one implies power series expansions in norm.

For real numbers $\alpha, \beta$,

$$
\alpha \vee \beta=\max \{\alpha, \beta\}, \quad \alpha \wedge \beta=\min \{\alpha, \beta .\} .
$$

\section{Standard Hilbert Spaces}

Given a faithful $\omega \in M_{*}^{+}$, we denote the associated GNS-vector by $\omega^{1 / 2}$ and identify the left and right GNS-spaces by the relation $\Delta_{\omega}^{1 / 2}\left(x \omega^{1 / 2}\right)=\omega^{1 / 2} x$, resulting in an $M$-bimodule $L^{2}(M, \omega)=\overline{M \omega^{1 / 2} M}$ with the positive cone $L^{2}(M, \omega)_{+}$and the compatible *-operation given by $L^{2}(M, \omega)_{+}=\overline{\left\{a \omega^{1 / 2} a^{*} ; a \in M\right\}}$ and $\left(a \omega^{1 / 2} b\right)^{*}=$ $b^{*} \omega^{1 / 2} a^{*}$ in such a way that these constitute a so-called standard form of $M$.

The dependence on $\omega$ as well as its faithfulness is then removed by the matrix ampliation technique: For each $\varphi \in M_{*}^{+}$, let $M \otimes \varphi^{1 / 2} \otimes M$ be a dummy of the algebraic tensor product $M \otimes M$, which is an $M$-bimodule in an obvious manner with a compatible ${ }^{*}$-operation defined by the relation $\left(a \otimes \varphi^{1 / 2} \otimes b\right)^{*}=b^{*} \otimes \varphi^{1 / 2} \otimes a^{*}$. On the algebraic direct sum

$$
\bigoplus_{\varphi \in M_{*}^{+}} M \otimes \varphi^{1 / 2} \otimes M
$$


of these ${ }^{*}$-bimodules, introduce a sesquiliear form by

$$
\begin{aligned}
\left(\bigoplus_{j=1}^{n} x_{j} \otimes \omega_{j}^{1 / 2} \otimes y_{j} \mid \bigoplus_{k=1}^{n} x_{k}^{\prime} \otimes \omega_{k}^{1 / 2} \otimes y_{k}^{\prime}\right) & \\
& =\sum_{j, k}\left(\left[\omega_{k}\right]\left(x_{k}^{\prime}\right)^{*} x_{j} \omega_{j}^{1 / 2} \mid \omega_{k}^{1 / 2} y_{k}^{\prime} y_{j}^{*}\left[\omega_{j}\right]\right)
\end{aligned}
$$

which is positive because of

$$
\begin{aligned}
\sum_{j, k}\left(\left[\omega_{k}\right] x_{k}^{*} x_{j} \omega_{j}^{1 / 2} \mid \omega_{k}^{1 / 2} y_{k} y_{j}^{*}\left[\omega_{j}\right]\right) & =\left(X \omega^{1 / 2} \mid \omega^{1 / 2} Y\right) \\
& =\left(X^{1 / 2} \omega^{1 / 2} Y^{1 / 2} \mid X^{1 / 2} \omega^{1 / 2} Y^{1 / 2}\right) \geq 0 .
\end{aligned}
$$

Here $\omega=\operatorname{diag}\left(\omega_{1}, \ldots, \omega_{n}\right)$ denotes a diagonal functional on the $n$-th matrix ampliation $M_{n}(M)$ of $M$ and

$$
X=[\omega]\left(\begin{array}{c}
x_{1}^{*} \\
\vdots \\
x_{n}^{*}
\end{array}\right)\left(\begin{array}{lll}
x_{1} & \ldots & x_{n}
\end{array}\right)[\omega] \text { and } Y=[\omega]\left(\begin{array}{c}
y_{1} \\
\vdots \\
y_{n}
\end{array}\right)\left(\begin{array}{lll}
y_{1}^{*} & \ldots & y_{n}^{*}
\end{array}\right)[\omega]
$$

are positive elments in $[\omega] M_{n}(M)[\omega]$. Recall that $[\omega]=\operatorname{diag}\left(\left[\omega_{1}\right], \ldots,\left[\omega_{n}\right]\right)$.

The associated Hilbert space is denoted by $L^{2}(M)$ and the image of $a \otimes \varphi^{1 / 2} \otimes b$ in $L^{2}(M)$ by $a \varphi^{1 / 2} b$. Here the notation is compatible with the one for $L^{2}(M, \varphi)$ because

$$
[\varphi] M[\varphi] \otimes \varphi^{1 / 2} \otimes[\varphi] M[\varphi] \ni a \otimes \varphi^{1 / 2} \otimes b \mapsto a \varphi^{1 / 2} b \in L^{2}(M, \varphi)
$$

gives an isometric map by the very definition of inner products. Similar remarks are in order for left and right GNS spaces.

The left and right actions of $M$ are compatible with taking quotients and they are bounded on $L^{2}(M)$ : For $a \in M$,

$$
\left\|\bigoplus_{j} a x_{j} \otimes \omega_{j}^{1 / 2} \otimes y_{j}\right\|^{2}=\left(\omega^{1 / 2} \mid Z J Y J \omega^{1 / 2}\right)
$$

with

$$
0 \leq Z=[\omega]\left(\begin{array}{c}
x_{1}^{*} \\
\vdots \\
x_{n}^{*}
\end{array}\right) a^{*} a\left(x_{1} \quad \ldots \quad x_{n}\right)[\omega] \leq\|a\|^{2} X
$$

Moreover, these actions give ${ }^{*}$-representations of $M:(a \xi \mid \eta)=\left(\xi \mid a^{*} \eta\right)$ and $(\xi a \mid \eta)=$ $\left(\xi \mid \eta a^{*}\right)$ for $\xi, \eta \in L^{2}(M)$ and $a \in M$, which is immediate from the definition of inner product. 
The *-operation on $L^{2}(M)$ is also compatible with the inner product:

$$
\begin{aligned}
\left\|\left(\bigoplus_{j} x_{j} \otimes \omega_{j}^{1 / 2} \otimes y_{j}\right)^{*}\right\|^{2} & =\left\|\bigoplus_{j} y_{j}^{*} \otimes \omega_{j}^{1 / 2} \otimes x_{j}^{*}\right\|^{2}=\left(Y \omega^{1 / 2} \mid \omega^{1 / 2} X\right) \\
& =\left(\left(\omega^{1 / 2} X\right)^{*} \mid\left(Y \omega^{1 / 2}\right)^{*}\right)=\left(X \omega^{1 / 2} \mid \omega^{1 / 2} Y\right) \\
& =\left\|\bigoplus_{j} x_{j} \otimes \omega_{j}^{1 / 2} \otimes y_{j}\right\|^{2} .
\end{aligned}
$$

In this way, we have constructed a ${ }^{*}$-bimodule $L^{2}(M)$ of $M$ in such a way that $L^{2}(M, \varphi) \subset L^{2}(M)$ for each $\varphi \in M_{*}^{+}$and the closed subspaces $\overline{M \varphi^{1 / 2}}, \overline{\varphi^{1 / 2} M}$ in $L^{2}(M)$ are naturally identified with the left and right GNS spaces of $\varphi$ respectively. Moreover, for $\varphi, \psi \in M_{*}^{+}$, we have $[\varphi] \overline{M \psi^{1 / 2}}=\overline{\varphi^{1 / 2} M}[\psi]$ in $L^{2}(M)$, which is just a reflection of the fact that the same identification inside $L^{2}\left(M_{n}(M), \omega\right)$ is used in the definition of inner product.

\section{Modular Algebras}

Recall the definition of (boundary) modular algebra which was introduced in 10 . to 'resolve' various cocycle relations in modular theory. We shall here describe it without essential use of the notion of weights.

Let $M$ be a $\mathrm{W}^{*}$-algebra which is assumed to admit a faithful $\omega \in M_{*}^{+}$for the moment. The modular algebra $M(i \mathbb{R})$ of $M$ is then the ${ }^{*}$-algebra generated by elements in $M$ and symbols $\varphi^{i t}$ for $\varphi \in M_{*}^{+}$and $t \in \mathbb{R}$ under the conditions that (i) $M(i \mathbb{R})$ contains $M$ as a ${ }^{*}$-subalgebra, (ii) $\left\{\varphi^{i t}\right\}_{t \in \mathbb{R}}$ is a one-parameter group of partial isometries satisfying $\varphi^{i 0}=[\varphi]$, (iii) $\varphi^{i t} a=\sigma_{t}^{\varphi, \psi}(a) \psi^{i t}$ for $\varphi, \psi \in M_{*}^{+}$, $a \in[\varphi] M[\psi]$ and $t \in \mathbb{R}$.

By utilizing a faithful $\omega \in M_{*}^{+}$, it turns out that $M(i \mathbb{R})$ is *-isomorphic to the algebraic crossed product of $M$ by $\left\{\sigma_{t}^{\omega}\right\}$ and therefore $M(i \mathbb{R})$ is an algebraic direct sum of $M(i t)=M \omega^{i t}=\omega^{i t} M$, where $M(i t)=\sum_{\varphi \in M_{*}^{+}} M \varphi^{i t} M$.

Thus the modular algebra $M(i \mathbb{R})$ is $i \mathbb{R}$-graded in the sense that $M(i t)^{*}=$ $M(-i t), M(i s) M(i t)=M(i(s+t))$ and $M(i 0)=M$.

We say that an element $a \in M$ is finitely supported if $a=[\varphi] a[\varphi]$ for some $\varphi \in M_{*}^{+}$. Let $M_{f}$ be the set of finitely supported elements in $M$.

Lemma 2.1. $M_{f}$ is a $\mathrm{w}^{*}$-dense ${ }^{*}$-subalgebra of $M$ and closed under sequential $\mathrm{w}^{*}$-limits in $M$. Moreover,

$$
M_{f}=\sum_{\varphi \in M_{*}^{+}} M[\varphi]=\sum_{\varphi \in M_{*}^{+}}[\varphi] M .
$$

Proof. Clearly $M_{f}$ is closed under the ${ }^{*}$-operation and $M_{f}$ is a subalgebra in view of $[\varphi] \vee[\psi] \leq[\varphi+\psi]$. The ${ }^{*}$-subalgebra $M_{f}$ is then $\mathrm{w}^{*}$-dense in $M$ in view of $\vee_{\varphi \in M_{*}^{+}}[\varphi]=1$. If $a=a[\varphi],\left[a \varphi a^{*}\right]$ is the left support of $a$ and $\left[\varphi+a \varphi a^{*}\right] a\left[\varphi a \varphi a^{*}\right]=$ $a$. Let $a$ be a ${ }^{*}$-limit of $\left\{a_{n}\right\}_{n \geq 1}$ in $M_{f}$ with $\left[\varphi_{n}\right]\left[a_{n}\right]\left[\varphi_{n}\right]=a_{n}$ for $n \geq 1$. Then, for $\varphi=\sum_{n=1}^{\infty} 2^{-n} \varphi_{n} / \varphi_{n}(1) \in M_{*}^{+},[\varphi] a[\varphi]$. 
Now we relax the existence of faithful functionals in $M_{*}^{+}$and set

$$
M_{f}(i \mathbb{R})=\bigcup_{\varphi \in M_{*}^{+}}[\varphi] M[\varphi](i \mathbb{R}),
$$

where the natural inclusions $[\varphi] M[\varphi](i \mathbb{R}) \subset[\psi] M[\psi](i \mathbb{R})$ for $\varphi, \psi \in M_{*}^{+}$satisfying $[\varphi] \subset[\psi]$ are assumed in the union.

Finally we add formal expressions of the form $\omega^{i t}=\sum_{j \in I} \omega_{j}^{i t}$ for families $\left\{\omega_{j} \in\right.$ $\left.M_{*}^{+}\right\}_{j \in I}$ of mutually orthogonal supports and allow products with elements in $M$ to get $\{M(i t)\}_{t \in \mathbb{R}}$ so that $M_{f}(i t) \subset M(i t)$ and $M(0)=M$. In what follows, a formal sum $\omega=\sum_{j \in I} \omega_{j}$ is referred to as a weight of $M$. A weight $\omega=\sum \omega_{j}$ is said to be faithful if $1=\sum\left[\omega_{j}\right]$ in $M$. Note that any weight is extended to a faithful one and $\left\{\omega^{i t}\right\}$ is a one-parameter group of unitaries in $M(i \mathbb{R})=\oplus M(i t)$ for a faithful $\omega$ and, for another choice of a faithful weight $\phi=\sum_{k \in J} \phi_{k}$ and $a \in M$,

$$
\phi^{i t} a \omega^{-i t}=\sum_{j, k} \phi_{k}^{i t} a \omega_{j}^{-i t}
$$

defines a continuous family of elements in $M$ so that it consists of unitaries when $a=1$ and $\sigma_{t}^{\omega}(a)=\omega^{i t} a \omega^{-i t}$ gives an automorphic action of $\mathbb{R}$ on $M$.

Remark 1 . Here weights are introduced in a formal and restricted way.

At this stage, we introduce two more classes of modular algebraic stuffs:

$$
M(i \mathbb{R}+1 / 2)=\sum_{t \in \mathbb{R}} M(i t+1 / 2), \quad M(i \mathbb{R}+1)=\sum_{t \in \mathbb{R}} M(i t+1),
$$

with

$$
M(i t+1 / 2)=\sum_{\varphi \in M_{*}^{+}} M \varphi^{i t+1 / 2}=\sum_{\varphi \in M_{*}^{+}} \varphi^{i t+1 / 2} M
$$

and

$$
M(i t+1)=\sum_{\varphi \in M_{*}^{+}} M \varphi^{i t+1}=\sum_{\varphi \in M_{*}^{+}} \varphi^{i t+1} M
$$

so that $M(1 / 2)=L^{2}(M)$ and $M(1)=M_{*}$.

These are $i \mathbb{R}$-graded ${ }^{*}$-bimodules of $M(i \mathbb{R})$ in an obvious way and we have a natural module map $M(i \mathbb{R}+1 / 2) \otimes_{M(i \mathbb{R})} M(i \mathbb{R}+1 / 2) \rightarrow M(i \mathbb{R}+1)$ which respects the grading in the sense that $M(i s+1 / 2) M(i t+1 / 2)=M(i(s+t)+1)$ for $s, t \in \mathbb{R}$. In particular, given a weight $\omega$ on $M$, we have $M(i t+s)=M(s) \omega^{i t}=\omega^{i t} M(s)$ for $s=1 / 2,1$.

The evaluation of $\varphi \in M_{*}$ at the unit $1 \in M$ is called the expectation of $\varphi$ and denoted by $\langle\varphi\rangle$. Note that the expectation satisfies the trace property for various combinations of multiplications such as $\langle a \varphi\rangle=\langle\varphi a\rangle$ and $\left\langle\varphi^{i t} \xi \psi^{-i t} \eta\right\rangle=\left\langle\psi^{-i t} \eta \varphi^{i t} \xi\right\rangle$ for $a \in M, \varphi, \psi \in M_{*}^{+}$and $\xi, \eta \in L^{2}(M)$.

The scaling $\varphi \mapsto e^{-s} \varphi$ on $M_{*}^{+}$gives rise to a *automorphic action $\theta_{s}$ of $s \in$ $\mathbb{R}$ (called the scaling automorphisms) on these modular stuffs: $\theta_{s}\left(x \varphi^{i t+r}\right)=$ $e^{-i s t-s r} x \varphi^{i t+r}$ for $x \in M, r \in\{0,1 / 2,1\}$ and $t \in \mathbb{R}$.

Remark 2. Since elements in $L^{2}(M)$ and $M_{*}$ are always 'finitely supported', we can decribe $M(i t+s)(s=1 / 2,1)$ without referring to weights. 


\section{Analytic Properties}

We here collect well-known analytic properties of modular stuffs (proofs can be found in [1] and [8] for example).

Lemma 3.1 (Modular Extension). For $\varphi, \psi \in M_{*}^{+}$and $a \in M, \mathbb{R} \ni t \mapsto \varphi^{i t} a \psi^{1-i t} \in$ $M_{*}$ is extended analytically to a norm-continuous function $\varphi^{i z} a \psi^{1-i z}$ on the strip $-1 \leq \operatorname{Im} z \leq 0$ with a bound

$$
\left\|\varphi^{i t+r} a \psi^{-i t+1-r}\right\| \leq\|\varphi a\|^{r}\|a \psi\|^{1-r} \quad(0 \leq r \leq 1)
$$

in such a way that

$$
\left.\left(\varphi^{i z} a \psi^{1-i z}\right)\right|_{z=t-i}=\varphi^{1+i t} a \psi^{-i t},\left.\quad\left(\varphi^{i z} a \psi^{1-i z}\right)\right|_{z=t-i / 2}=\varphi^{i t+1 / 2} a \psi^{-i t+1 / 2} .
$$

Corollary 3.2 (KMS condition). Let $\varphi, \psi \in M_{+}^{*}$ and $a \in[\varphi] M[\psi]$. Then the function $\sigma_{t}^{\varphi, \psi}(a) \psi^{1 / 2}=\varphi^{i t} a \psi^{-i t} \psi^{1 / 2}$ of $t \in \mathbb{R}$ is analytically extended to an $L^{2}(M)$ valued continuous function $\varphi^{i z} a \psi^{-i z+1 / 2}$ of $z \in \mathbb{R}-i[0,1 / 2]$ so that $\left(\varphi^{i z} a \psi^{-i z+1 / 2}\right)_{z=t-i / 2}=$ $\varphi^{1 / 2} \varphi^{i t} a \psi^{-i t}=\varphi^{1 / 2} \sigma_{t}^{\varphi, \psi}(a)$.

Lemma 3.3. Let $\omega \in M_{*}^{+}$be faithful and let $a \in M$. Then the following conditions are equivalent.

(i) The inequality $a^{*} \omega a \leq \omega$ holds in $M_{*}^{+}$.

(ii) We can find a function $a(z) \in M$ of $z \in \mathbb{R}-i[0,1 / 2]$ such that $a(t)=$ $\omega^{i t} a \omega^{-i t}$ for $t \in \mathbb{R}, a(z) \xi \in L^{2}(M)$ is norm-analytic in $z$ for any $\xi \in L^{2}(M)$ and $\|a(-i / 2)\| \leq 1$.

(iii) We can find an element $b \in M$ satisfying $\|b\| \leq 1$ and $\omega^{1 / 2} a=b \omega^{1 / 2}$.

Moreover, if this is the case, with the notation in (ii), $\xi a(z) \in L^{2}(M)$ is normcontinuous in $z$ for every $\xi \in L^{2}(M)$.

Corollary 3.4. For $\varphi, \psi \in M_{*}^{+}$, the following conditions are equivalent.

(i) The inequality $\varphi \leq \psi$ holds in $M_{*}^{+}$.

(ii) $[\varphi] \leq[\psi]$ and the function $\varphi^{i t} \psi^{-i t}$ of $t \in \mathbb{R}$ is analytically extended to an $M$-valued function $\varphi^{i z} \psi^{-i z}$ of $z \in \mathbb{R}-i[0,1 / 2]$ so that $\varphi^{i z} \psi^{-i z} \xi \in L^{2}(M)$ is norm-continuous in $z$ for any $\xi \in L^{2}(M)$ and $\left\|\varphi^{1 / 2} \psi^{-1 / 2}\right\| \leq 1$.

(iii) We can find an element $c \in M$ satisfying $\|c\| \leq 1$ and $\varphi^{1 / 2}=c \psi^{1 / 2}$.

Moreover, if this is the case, $\xi \varphi^{i z} \psi^{-i z} \in L^{2}(M)$ is norm-continuous in $z \in \mathbb{R}-$ $i[0,1 / 2]$ for any $\xi \in L^{2}(M)$.

Remark 3. Under the above majorization conditions, the relevant analytic extensions are norm-bounded as $M$-valued functions of $z \in \mathbb{R}-i[0,1 / 2]$ thanks to BanachSteinhaus theorem.

\section{Sectional Continuity}

We now describe continuity properties of families $\{M(i t+r)\}_{t \in \mathbb{R}}$ for $r=0,1 / 2$ and 1 . Let us begin with a simple observation on the continuity of modular actions: Let $\varphi=\sum \varphi_{j}$ and $\psi=\sum \psi_{k}$ be weights on $M$ in our sense. For $\xi \in L^{2}(M)$,

$$
\varphi^{i t} \xi \psi^{-i t}=\sum_{j, k} \varphi_{j}^{i t} \xi \psi_{k}^{-i t}
$$


is norm-continuous in $t \in \mathbb{R}$ as an orthogonal sum of $L^{2}(M)$-valued norm-continuous functions $\varphi_{j}^{i t} \xi \psi_{k}^{-i t}$. As any $\phi \in M_{*}^{+}$has an expression $\xi \eta$ with $\xi, \eta \in L^{2}(M)$, one sees that

$$
\varphi^{i t} \phi \psi^{-i t}=\left(\varphi^{i t} \xi \omega^{-i t}\right)\left(\omega^{i t} \eta \psi^{-i t}\right)
$$

( $\omega$ being an auxiliary faithful weight) is an $M_{*}$-valued norm-continuous function of $t \in \mathbb{R}$ as a product of $L^{2}(M)$-valued norm-continuous functions.

The following facts on continuity of sections of $\{M(i t+r)\}$ are then more or less straightforward from this observation.

Lemma 4.1. For a section $x=\{x(t)\}$ of $\{M(i t)\}$, the following conditions are equivalent.

(i) There exists a faithful weight $\omega$ on $M$ such that $\omega^{-i t} x(t) \in M$ is $\mathrm{w}^{*}$ continuous in $t \in \mathbb{R}$.

(ii) There exists a faithful weight $\omega$ on $M$ such that $x(t) \omega^{-i t} \in M$ is $\mathrm{w}^{*}$ continuous in $t \in \mathbb{R}$.

(iii) For any faithful weight $\omega$ on $M, \omega^{-i t} x(t) \in M$ is $\mathrm{w}^{*}$-continuous in $t \in \mathbb{R}$.

(iv) For any faithful weight $\omega$ on $M, x(t) \omega^{-i t} \in M$ is $\mathrm{w}^{*}$-continuous in $t \in \mathbb{R}$.

(v) For any $\phi \in M_{*}^{+}, \phi^{-i t} x(t) \in M$ is $\mathrm{w}^{*}$-continuous in $t \in \mathbb{R}$.

(vi) For any $\phi \in M_{*}^{+}, x(t) \phi^{-i t} \in M$ is $\mathrm{w}^{*}$-continuous in $t \in \mathbb{R}$.

Moreover, if $\{x(t)\}$ satisfies these equivalent conditions, $\|x(t)\|$ is locally bounded in $t \in \mathbb{R}$.

We say that a section $\{x(t)\}$ is $\mathbf{w}^{*}$-continuous if it satisfies any of these equivalent conditions.

We here introduce the ${ }^{*}$-operation on sections by

$$
x^{*}(t)=x(-t)^{*} \in M(i t+s) \text { for a section }\{x(t) \in M(i t+r)\} .
$$

As a consequence of the above lemma, for a section $x(t) \in M(i t), x^{*}(t)$ as well as $a x(t) b$ with $a, b \in M$ are $\mathrm{w}^{*}$-continuous if so is $x(t)$.

Lemma 4.2. Let $p=1$ or 2 with the notation $L^{1}(M)=M_{*}$ for $p=1$. Then the following conditions on a section $\{\xi(t)\}$ of $\{M(i t+1 / p)\}$ are equivalent.

(i) There exists a faithful weight $\omega$ on $M$ such that $\omega^{-i t} \xi(t) \in L^{p}(M)$ is norm-continuous in $t \in \mathbb{R}$.

(ii) There exists a faithful weight $\omega$ on $M$ such that $\xi(t) \omega^{-i t} \in L^{p}(M)$ is norm-continuous in $t \in \mathbb{R}$.

(iii) For any faithful weight $\omega$ on $M, \omega^{-i t} \xi(t) \in L^{p}(M)$ is norm-continuous in $t \in \mathbb{R}$.

(iv) For any faithful weight $\omega$ on $M, \xi(t) \omega^{-i t} \in L^{p}(M)$ is norm-continuous in $t \in \mathbb{R}$.

(v) For any $\phi \in M_{*}^{+}, \phi^{-i t} \xi(t) \in L^{p}(M)$ is norm-continuous in $t \in \mathbb{R}$.

(vi) For any $\phi \in M_{*}^{+}, \xi(t) \phi^{-i t} \in L^{p}(M)$ is norm-continuous in $t \in \mathbb{R}$.

We say that a section $\{\xi(t)\}$ is norm-continuous if it satisfies any of these equivalent conditions. Notice here that $\xi^{*}(t)=\xi(-t)^{*}$ is norm-continuous if so is $\xi(t)$.

Definition 4.3. A section $\{x(t)\}$ of $\{M(i t)\}_{t \in \mathbb{R}}$ is said to be $\mathbf{s}^{*}$-continuous if $x(t) \xi$ and $\xi x(t)$ are norm-continuous for any $\xi \in L^{2}(M)$. Notice that $x^{*}(t)$ is $\mathrm{s}^{*}$ continuous if and only if so is $x(t)$ in view of $x^{*}(t) \xi=\left(\xi^{*} x(-t)\right)^{*}$ and $\xi x^{*}(t)=$ $\left(x(-t) \xi^{*}\right)^{*}$. Clearly $\mathrm{s}^{*}$-continuous sections are $\mathrm{w}^{*}$-continuous. 
To control the norm of a $\mathrm{w}^{*}$-continuous section $x=\{x(t) \in M(i t)\}$, two norms are introduced by

$$
\|x\|_{\infty}=\sup \{\|x(t)\| ; t \in \mathbb{R}\}, \quad\|x\|_{1}=\int_{\mathbb{R}}\|x(t)\| d t
$$

and $x(t)$ is said to be bounded if $\|x\|_{\infty}<\infty$ and integrable if $\|x\|_{1}<\infty$. Note here that $\|x(t)\|$ is locally bounded and lower-semicontinuous.

Lemma 4.4. The following conditions on a section $\{x(t)\}$ of $\{M(i t)\}$ are equivalent.

(i) For any $\xi \in L^{2}(M),\{x(t) \xi\}$ is a norm-continuous section of $\{M(i t+1 / 2)\}$.

(ii) For any $\varphi \in M_{*}^{+}$and any $\xi \in L^{2}(M), x(t) \varphi^{-i t} \xi \in L^{2}(M)$ is normcontinuous in $t \in \mathbb{R}$.

(iii) The norm function $\|x(t)\|$ is locally bounded and, for a sufficiently large $\phi \in M_{*}^{+}, x(t) \phi^{-i t+1 / 2} \in L^{2}(M)$ is norm-continuous in $t \in \mathbb{R}$, i.e., given any $\varphi \in M_{*}^{+}$, we can find $\phi \in M_{*}^{+}$such that $\varphi \leq \phi$ and $x(t) \phi^{-i t+1 / 2} \in L^{2}(M)$ is norm-continuous in $t \in \mathbb{R}$.

Corollary 4.5. A section $x(t) \in M(i t)$ is $\mathrm{s}^{*}$-continuous if and only if $\|x(t)\|$ is locally bounded and $L^{2}(M)$-valued functions $x(t) \phi^{-i t+1 / 2}, \phi^{-i t+1 / 2} x(t)$ are normcontinuous for a sufficiently large $\phi \in M_{*}^{+}$.

A section $\{x(t) \in M(i t)\}_{t \in \mathbb{R}}$ is said to be finitely supported if we can find $\phi \in M_{*}^{+}$so that $x(t)=[\phi] x(t)[\phi]$ for every $t \in \mathbb{R}$. We say that $\{x(t)\}$ is locally bounded (bounded) if so is the function $\|x(t)\|$ of $t$.

\section{Convolution Algebra}

Consider a bounded, $\mathrm{s}^{*}$-continuous and integrable section $\{f(t) \in M(i t)\}$ and identify it with a formal expression like $\int_{\mathbb{R}} f(t) d t$, which is compatible with the *-operation by

$$
\left(\int_{\mathbb{R}} f(t) d t\right)^{*}=\int_{\mathbb{R}} f(t)^{*} d t=\int_{\mathbb{R}} f(-t)^{*} d t=\int_{\mathbb{R}} f^{*}(t) d t .
$$

Moreover, a formal rewriting

$$
\int_{\mathbb{R}} f(s) d s \int_{\mathbb{R}} g(t) d t=\int_{\mathbb{R}}\left(\int_{\mathbb{R}} f(s) g(t-s) d s\right) d t
$$

suggests to define a product of $f$ and $g$ by

$$
(f g)(t)=\int_{\mathbb{R}} f(s) g(t-s) d s=\int_{\mathbb{R}} f(t-s) g(s) d s .
$$

It is then a routine work to check that the totality of such sections constitutes a normed *-algebra in such a way that

$$
\|f g\|_{\infty} \leq\left(\|f\|_{1}\|g\|_{\infty}\right) \wedge\left(\|f\|_{\infty}\|g\|_{1}\right), \quad\|f g\|_{1} \leq\|f\|_{1}\|g\|_{1} .
$$

We notice that the scaling automorphism $\theta_{s}$ on $\{M(i t)\}$ induces a *-automorphic action on the ${ }^{*}$-algebra of sections by $\left(\theta_{s} f\right)(t)=e^{-i s t} f(t)$.

Here we shall apply formal arguments to illustrate how tracial functionals can be associated to this kind of *-algebras. 
Formal manipulation is an easy business: Imagine that a section $f(t)$ has an analytic extension to the region $-1 \leq \operatorname{Im} z \leq 0$ in some sense so that $f^{*}(z)=$ $f(-\bar{z})^{*}$ and define a linear functional by

$$
\tau\left(\int_{\mathbb{R}} f(t) d t\right)=\langle f(-i)\rangle .
$$

Note that $f(-i)$ in the right hand side belongs to $M(1)=M_{*}$. We then have

$$
\begin{aligned}
\tau\left(f^{*} f\right) & =\int_{\mathbb{R}}\left\langle f^{*}(s) f(-i-s)\right\rangle d s=\int_{\mathbb{R}}\left\langle f^{*}(s-i / 2) f(-s-i / 2)\right\rangle d s \\
& =\int_{\mathbb{R}}\left\langle f(-s-i / 2)^{*} f(-s-i / 2)\right\rangle d s \geq 0,
\end{aligned}
$$

where Cauchy's integral theorem is formally used in the first line. The trace property is seen from

$$
\begin{aligned}
\tau(f g) & =\int\langle f(s) g(-i-s)\rangle d s=\int\langle f(t-i) g(-t)\rangle d t \\
& =\int\langle g(-t) f(t-i)\rangle d t=\int\langle g(t) f(-t-i)\rangle d t=\tau(g f),
\end{aligned}
$$

where Cauchy's integral theorem is again used formally in the first line.

Going back to the sane track, it turns out that it is not easy to make all of the above formal arguments rigorous at least in a reference-weight-free fashion. Instead we shall construct a Hilbert algebra as a halfway business in what follows, which is enough to extract the tracial functional.

\section{Hilbert Algebras}

Definition 6.1. A section $\{f(t) \in M(i t)\}$ is said to be half-analytic if, for a sufficiently large $\phi \in M_{*}^{+}$, the function $f_{\phi}\left(t^{\prime}, t^{\prime \prime}\right)=\phi^{-i t^{\prime}} f\left(t^{\prime}+t^{\prime \prime}\right) \phi^{-i t^{\prime \prime}}$ of $\left(t^{\prime}, t^{\prime \prime}\right) \in \mathbb{R}^{2}$ is analytically extended to a bounded $M$-valued s*-continuous function $f_{\phi}\left(z^{\prime}, z^{\prime \prime}\right)=\phi^{-i z^{\prime}} f\left(z^{\prime}+z^{\prime \prime}\right) \phi^{-i z^{\prime \prime}}$ of $\left(z^{\prime}, z^{\prime \prime}\right) \in T_{[0,1 / 2]}$.

Note here that sufficient largeness in the condition has a meaning: For a $\phi$ majorized by $\omega \in M_{*}^{+}, \phi^{i t} \omega^{-i t}$ is analytically extended to a $\mathrm{s}^{*}$-continuous function $\phi^{i z} \omega^{-i z}$ of $z \in \mathbb{R}-i[0,1 / 2]$ (Corollary [3.4) and therefore $\omega^{-i t^{\prime}} f\left(t^{\prime}+t^{\prime \prime}\right) \omega^{-i t^{\prime \prime}}$ has an analytic extension of the form $\left(\omega^{-i z^{\prime}} \phi^{i z^{\prime}}\right)\left(\phi^{-i z^{\prime}} f\left(z^{\prime}+z^{\prime \prime}\right) \phi^{-i z^{\prime \prime}}\right)\left(\phi^{i z^{\prime \prime}} \omega^{-i z^{\prime \prime}}\right)$, which is $s^{*}$-continuous as a product of $s^{*}$-continuous locally bounded operator-valued functions.

Note also that the s*-continuity of $\phi^{-i z^{\prime}} f\left(z^{\prime}+z^{\prime \prime}\right) \phi^{-i z^{\prime \prime}}$ is equivalent to the normcontinuity of $L^{2}$-valued functions $\left(\phi^{-i z^{\prime}} f\left(z^{\prime}+z^{\prime \prime}\right) \phi^{-i z^{\prime \prime}}\right) \phi^{1 / 2}$ and $\phi^{1 / 2}\left(\phi^{-i z^{\prime}} f\left(z^{\prime}+\right.\right.$ $\left.z^{\prime \prime}\right) \phi^{-i z^{\prime \prime}}$ ) (Lemma 4.4). These are analytic extensions of $\phi^{-i t^{\prime}} f\left(t^{\prime}+t^{\prime \prime}\right) \phi^{1 / 2-i t^{\prime \prime}}$ and $\phi^{1 / 2-i t^{\prime}} f\left(t^{\prime}+t^{\prime \prime}\right) \phi^{-i t^{\prime \prime}}$, whence simply denoted by $\phi^{-i z^{\prime}} f\left(z^{\prime}+z^{\prime \prime}\right) \phi^{1 / 2-i z^{\prime \prime}}$ and $\phi^{1 / 2-i z^{\prime}} f\left(z^{\prime}+z^{\prime \prime}\right) \phi^{-i z^{\prime \prime}}$ respectively.

Warning: No separate meaning of $f(z)$ is assigned here.

It is immediate to see that $f(t)$ is half-analytic if and only if so is $f^{*}(t)=f(-t)^{*}$ in such a way that

$$
\phi^{-i z^{\prime}} f^{*}\left(z^{\prime}+z^{\prime \prime}\right) \phi^{-i z^{\prime \prime}}=\left(\phi^{i \overline{z^{\prime \prime}}} f\left(-\overline{z^{\prime \prime}}-\overline{z^{\prime}}\right) \phi^{i \overline{z^{\prime}}}\right)^{*} .
$$


To get the convolution product in a manageable way, we impose the following decaying condition. For a half-analytic section $f(t) \in M(i t)$, the obvious identity $f_{\phi}\left(z^{\prime}+s^{\prime}, z^{\prime \prime}+s^{\prime \prime}\right)=\phi^{-i s^{\prime}} f_{\phi}\left(z^{\prime}, z^{\prime \prime}\right) \phi^{-i s^{\prime \prime}}$ shows that $\left\|f_{\phi}\left(z^{\prime}, z^{\prime \prime}\right)\right\|$ depends only on $r^{\prime}=-\operatorname{Im} z^{\prime}, r^{\prime \prime}=-\operatorname{Im} z^{\prime \prime}$ and $t=\operatorname{Re}\left(z^{\prime}+z^{\prime \prime}\right)$, which enables us to introduce

$$
|f|_{\phi}(t)=\sup \left\{\left\|f_{\phi}\left(z^{\prime}, z^{\prime \prime}\right)\right\| ; r^{\prime} \geq 0, r^{\prime \prime} \geq 0, r^{\prime}+r^{\prime \prime} \leq 1 / 2\right\} .
$$

A half-analytic section $f(t)$ is said to be of Gaussian decay if, for a sufficiently large $\phi \in M_{*}^{+}$, we can find $\delta>0$ so that $|f|_{\phi}(t)=O\left(e^{-\delta t^{2}}\right)$.

Now let $\mathcal{N}$ be the vector space of half-analytic sections of Gaussian decay, which is closed under taking the *-operation by (1). It is immediate to see that the scaling automorphisms leave $\mathcal{N}$ invariant so that $\phi^{-i z^{\prime}}\left(\theta_{s} f\right)\left(z^{\prime}+z^{\prime \prime}\right) \phi^{-i z^{\prime \prime}}=$ $e^{-i s\left(z^{\prime}+z^{\prime \prime}\right)} \phi^{-i z^{\prime}} f\left(z^{\prime}+z^{\prime \prime}\right) \phi^{-i z^{\prime \prime}}$.

Let $f, g \in \mathcal{N}$. Thanks to the Gaussian decay assumption, the convolution product $f g$ has a meaning and $(f g)(t)$ is an $\mathrm{s}^{*}$-continuous section. To see $f g \in \mathcal{N}$, we therefore need to check that it admits a half-analytic extension of Gaussian decay.

Choose an auxiliary weight $\omega$ which supports both $f$ and $g$. Then

$$
\begin{aligned}
\phi^{-i z^{\prime}}\left((f g)\left(z^{\prime}+z^{\prime \prime}\right) \phi^{-i z^{\prime \prime}}\right) & \\
& =\int_{\mathbb{R}} \phi^{-i z^{\prime}} f\left(z^{\prime}+s\right) \omega^{-i s} \sigma_{s}^{\omega}\left(g\left(z^{\prime \prime}-s\right) \phi^{-i\left(z^{\prime \prime}-s\right)}\right) \omega^{i s} \phi^{-i s} d s
\end{aligned}
$$

gives the $\mathrm{s}^{*}$-continuous analytic extension with its norm estimated by

$$
\begin{aligned}
\left\|\phi^{-i z^{\prime}}(f g)\left(z^{\prime}+z^{\prime \prime}\right) \phi^{-i z^{\prime \prime}}\right\| & \\
& \leq \int_{\mathbb{R}}|f|_{\phi}\left(\operatorname{Re} z^{\prime}+s\right)|g|_{\phi}\left(\operatorname{Re} z^{\prime \prime}-s\right) d s=O\left(e^{-\epsilon \delta t^{2} /(\epsilon+\delta)}\right)
\end{aligned}
$$

for $t=\operatorname{Re}\left(z^{\prime}+z^{\prime \prime}\right)$ if $|f|_{\phi}(t)=O\left(e^{-\epsilon t^{2}}\right)$ and $|g|_{\phi}(t)=O\left(e^{-\delta t^{2}}\right)$.

So far $\mathcal{N}$ is shown to be a ${ }^{*}$-algebra with an automorphic action of $\mathbb{R}$ by scaling automorphisms. We next introduce an inner product which makes $\mathcal{N}$ into a Hilbert algebra.

Lemma 6.2. The following identity holds for $f \in \mathcal{N}$ and sufficiently large $\phi, \varphi \in$ $M_{*}^{+}$.

$$
[\varphi]\left(f(t-i / 2) \phi^{-i t-1 / 2}\right) \phi^{i t+1 / 2}=\varphi^{i t+1 / 2}\left(\varphi^{-i t-1 / 2} f(t-i / 2)\right)[\phi]
$$

(the left hand side is therefore depends only on $[\varphi]$ while the right hand side depends only on $[\phi]$ and the common element in $M(i t+1 / 2)$ is reasonably denoted by $[\varphi] f(t-i / 2)[\phi])$.

Proof. For $a \in M$, the identity

$$
\left\langle\left(f(t) \phi^{-i t}\right) \phi^{1 / 2} \sigma_{t}^{\phi, \varphi}(a) \varphi^{1 / 2}\right\rangle=\left\langle\varphi^{1 / 2}\left(\varphi^{-i t} f(t)\right) \phi^{1 / 2} a\right\rangle
$$

is analytically continued from $t$ to $t-i / 2$ to get

$$
\left\langle\left(f(t-i / 2) \phi^{-i t-1 / 2}\right) \phi^{1 / 2} \phi^{1 / 2} \sigma_{t}^{\phi, \varphi}(a)\right\rangle=\left\langle\varphi^{1 / 2}\left(\varphi^{-i t-1 / 2} f(t-i / 2)\right) \phi^{1 / 2} a\right\rangle
$$

(use the KMS-condition at $\sigma_{t}^{\phi, \varphi}(a) \varphi^{1 / 2}$ ) and, after a simple rewriting,

$$
\left\langle\left(f(t-i / 2) \phi^{-i t-1 / 2}\right) \phi^{i t+1 / 2} \phi^{1 / 2} a \varphi^{-i t}\right\rangle=\left\langle\varphi^{i t+1 / 2}\left(\varphi^{-i t-1 / 2} f(t-i / 2)\right) \phi^{1 / 2} a \varphi^{-i t}\right\rangle \text {. }
$$


Since $[\varphi] f(t-i / 2)[\phi]=[\varphi]\left(\left[\varphi^{\prime}\right] f(t-i / 2)\left[\phi^{\prime}\right]\right)[\phi]$ whenever $[\varphi] \leq\left[\varphi^{\prime}\right]$ and $[\phi] \leq\left[\phi^{\prime}\right]$, $\|[\varphi] f(t-i / 2)[\phi]$ is increasing in $[\varphi]$ and $[\phi]$. We claim that

$$
f(t-i / 2)=\lim _{\substack{[\varphi] \rightarrow 1 \\[\phi] \rightarrow 1}}[\varphi] f(t-i / 2)[\phi]
$$

exists in $M(i t+1 / 2)$. In fact, if not, we can find increasing sequences $\varphi_{n}$ and $\phi_{n}$ in $M_{*}^{+}$so that $\lim _{n \rightarrow \infty}\left\|\left[\varphi_{n}\right] f(t-i / 2)\left[\phi_{n}\right]\right\|=\infty$, which contradicts with

$$
\left\|\left[\varphi_{n}\right] f(t-i / 2)\left[\phi_{n}\right]\right\| \leq\|[\varphi] f(t-i / 2)[\phi]\|<\infty
$$

for the choice $\varphi=\sum \varphi_{n} / 2^{n}\left\|\varphi_{n}\right\|, \phi=\sum \phi_{n} / 2^{n}\left\|\phi_{n}\right\|$.

Moreover, the same reasoning reveals that we can find $\varphi, \phi \in M_{*}^{+}$so that $f(t-$ $i / 2)=[\varphi] f(t-i / 2)=f(t-i / 2)[\phi]$. Consequently, $\{f(t-i / 2) \in M(i t+1 / 2)\}$ is a norm-continuous section of Gaussian decay from the expression

$$
f(t-i / 2)=f(t-i / 2)[\phi]=\left(f(t-i / 2) \phi^{-i t-1 / 2}\right) \phi^{i t+1 / 2}
$$

which is valid for a sufficiently large $\phi$.

Remark 4. By an analytic continuation, one sees that any half-analytic section $\{f(t)\}$ of $\{M(i t)\}$ is finitely supported in the sense that there exists $\phi \in M_{*}^{+}$ satisfying $f(t)=[\phi] f(t)[\phi]$ for every $t \in \mathbb{R}$.

Example 6.3. Let $\varphi \in M_{*}^{+}$and $a, b \in[\varphi] M[\varphi]$ be entirely analytic for $\sigma_{t}^{\varphi}$. Then, for $\alpha>0$ and $\beta \in \mathbb{C}, f(t)=e^{-\alpha t^{2}+\beta t} a \varphi^{i t} b$ belongs to $\mathcal{N}$ and its boundary section is $f(t-i / 2)=e^{-\alpha(t-i / 2)^{2}+\beta(t-i / 2)} a \varphi^{i t+1 / 2} b$.

The inner product is now introduced by

$$
(f \mid g)=\int_{\mathbb{R}}(f(t-i / 2) \mid g(t-i / 2)) d t=\int_{\mathbb{R}}\left\langle f(t-i / 2)^{*} g(t-i / 2)\right\rangle d t,
$$

which is clearly positive-definite and the completed Hilbert space $\mathcal{H}$ is naturally identified with the direct integral

$$
\mathcal{H}=\oint_{\mathbb{R}} M(i t+1 / 2) d t
$$

because $\mathcal{N}$ provides a dense set of measurable sections in the right hand side. The Hilbert space $\mathcal{H}$ is then made into a *-bimodule of $M(i \mathbb{R})$ by

and

$$
a \omega^{i s} \oint_{\mathbb{R}} \xi(t) d t=\oint_{\mathbb{R}} a \omega^{i s} \xi(t-s) d t
$$

$$
(\oint \xi(t) d t)^{*}=\oint_{\mathbb{R}} \xi(-t)^{*} d t
$$

in such a way that actions of $M(i t)$ on $\mathcal{H}$ are $\mathrm{s}^{*}$-continuous.

Since the family $\{M(i t+1 / 2)\}$ is trivialized by obvious isomorphisms $L^{2}(M) \omega^{i t} \cong$ $L^{2}(M) \cong \omega^{i t} L^{2}(M)$ in terms of a faithful weight $\omega$ on $M$, we have identifications $\mathcal{H} \cong L^{2}(M) \otimes L^{2}(\mathbb{R})$ in two ways, which transforms left and right multiplications of $\omega^{i t}$ into a translational unitary by $t \in \mathbb{R}$. Recall that our weights are orthogonal direct sums of bounded functionals and the multiplication of $\omega^{i s}$ on $\mathcal{H}$ gives a continuous one-parameter group of unitaries.

With these observations in mind, it is immediate to check the axioms of Hilbert algebra: the left and right multiplications are bounded with respect to the inner product, $\mathcal{N}^{2}$ is dense in $\mathcal{H}$ and $\left(f^{*} \mid g^{*}\right)=(g \mid f)$ for $f, g \in \mathcal{N}$. 
Remark 5. Note that the scaling automorphism $\theta_{s}$ satisfies $\left(\theta_{s} f\right)(t-i / 2)=e^{-i s t-s / 2} f(t-$ $i / 2)$ and hence scales the inner product: $\left(\theta_{s} f \mid \theta_{s} g\right)=e^{-s}(f \mid g)$ for $f, g \in \mathcal{N}$.

In this way, we have constructed a Hilbert algebra $\mathcal{N}$. The associated von Neumann algebra is denoted by $N=M \rtimes \mathbb{R}$ and referred to as the Takesaki dual of $M$ in what follows. The scaling automorphisms $\theta_{s}$ of $\mathcal{N}$ induce a ${ }^{*}$-automorphic action (also denoted by $\left.\theta_{s}\right)$ of $\mathbb{R}$ on $N$ by $\theta_{s}(l(f))=l\left(\theta_{s} f\right)$, which is referred to as the dual action. Here $l(f)$ denotes a bounded operator on $\mathcal{H}$ defined by $l(f) g=f g$ for $g \in \mathcal{N}$.

Let $\omega$ be a faithful weight on $M$. From the convolution form realization of $\mathcal{N}$ on $\mathcal{H}$, one sees that $N$ contains $M$ as well as $\omega^{i t}$ as operators by left multiplication and these in turn generates $N$. Likewise right multiplications of $M$ and $\omega^{i t}$ generates the right action of $N$ on $\mathcal{H}$. Thus the Takesaki dual of $M$ is isomorphic to the crossed product of $M$ with respect to the modular automorphism group $\left\{\sigma_{t}^{\omega}\right\}$, which justifies our notation $M \rtimes \mathbb{R}$ for $N$.

We record here the following well-known fact for later use together with a proof to illustrate how the essence can be easily captured in the modular algebra formalism.

Theorem 6.4 (Takesaki). The fixed-point algebra $N^{\theta}$ of $N$ under the dual action $\theta$ is identified with $M$.

Proof. Through $\mathcal{H} \cong L^{2}(M) \otimes L^{2}(\mathbb{R})$ adapted to the trivialization $M(i t+1 / 2) \omega^{-i t}=$ $L^{2}(M)$ of $M(i t+1 / 2)$, the right action of $\omega^{i s}$ is realized on $L^{2}(\mathbb{R})$ by translations whereas $\theta_{s}$ by multiplication of $e^{-i s t}$ on $L^{2}(\mathbb{R})$. Since these generate $\mathcal{B}\left(L^{2}(\mathbb{R})\right)$ (Stone-von Neumann), $N^{\theta}$ is identified with $\left(\mathcal{B}\left(L^{2}(M)\right) \otimes 1\right) \cap \operatorname{End}\left(\mathcal{H}_{M}\right)$. Let $a \in M$ and $f, g \in L^{2}(\mathbb{R})$. For $\xi, \eta \in L^{2}(M)$,

$$
(\eta \otimes g \mid(\xi \otimes f) a)=\left(\eta \mid \xi \sigma_{\bar{g} f}^{\omega}\right) \quad \text { with } \quad \sigma_{\bar{g} f}^{\omega}=\int_{\mathbb{R}} \overline{g(t)} f(t) \omega^{i t} a \omega^{-i t} \in M
$$

shows that $T \in \mathcal{B}\left(L^{2}(M)\right)$ belongs to $N^{\theta}$ if and only if it is in the commutant of the right action of $\left\{\sigma_{h}^{\omega}(a) ; h \in L^{1}(\mathbb{R})\right\}$ on $L^{2}(M)$. Since $\left\{\sigma_{h}^{\omega}(a) ; a \in M, h \in L^{1}(\mathbb{R})\right\}$ generates $M$, this implies $N^{\theta} \subset M$.

Now we introduce some notations and conventions in connection with our Hilbert algebra: $\mathcal{N}$ is regarded as a ${ }^{*}$-subalgebra of $N$ and we write $\mathcal{N} \tau^{1 / 2}=\tau^{1 / 2} \mathcal{N}$ to indicate the corresponding subspace in $\mathcal{H}=\oint_{\mathbb{R}} M(i t+1 / 2) d t$, where $\tau^{1 / 2}$ is just a dummy symbol but its square $\tau$ will be soon identified with the standard trace on $N$. Thus $h \in \mathcal{N}$ is identified with an operator on $\mathcal{H}$ satisfying $h\left(f \tau^{1 / 2}\right)=(h f) \tau^{1 / 2}$ for $f \in \mathcal{N}$, whereas $f \tau^{1 / 2}=\tau^{1 / 2} f=\oint_{\mathbb{R}} f(t-i / 2) d t$.

Let $B \supset \mathcal{N}$ be a dense ${ }^{*}$-ideal of $N$ such that $B \tau^{1 / 2}=\tau^{1 / 2} B$ is the set of bounded vectors in $\mathcal{H} ; y \in N$ belongs to $B$ if and only if there exists a vector $\eta \in \mathcal{H}$ satisfying $\eta f=y\left(f \tau^{1 / 2}\right)=y\left(\tau^{1 / 2} f\right)$ for any $f \in \mathcal{N}$ and, if this is the case, we write $\eta=y \tau^{1 / 2}=\tau^{1 / 2} y$. Recall that the standard trace $\tau$ on $N_{+}$is defined by $\tau\left(y^{*} y\right)=\left(y \tau^{1 / 2} \mid y \tau^{1 / 2}\right)$ if $y \in B$ and $\tau\left(y^{*} y\right)=\infty$ otherwise. Note that, for $f, g \in \mathcal{N}$, $f^{*} g \in \mathcal{N}^{2}$ is in the trace class and its trace is calculated by

$$
\tau\left(f^{*} g\right)=\int_{\mathbb{R}}(f(t-i / 2) \mid g(t-i / 2)) d t=\left(f \tau^{1 / 2} \mid g \tau^{1 / 2}\right),
$$

which justifies our notation $f \tau^{1 / 2}$. 
From the scaling relation $\left(\theta_{s} f\right)(t-i / 2)=e^{-i s t-s / 2} f(t-i / 2)$, the inner product is scaled by a factor $e^{-s}$ under the *-automorphism of $\mathcal{N}$ and hence the associated trace $\tau$ scales like $\tau\left(\theta_{s}\left(y^{*} y\right)\right)=e^{-s} \tau\left(y^{*} y\right)$ for $y \in N$.

To each $\xi, \eta \in \mathcal{H}$, a sesquilinear element $\xi^{*} \eta \in N_{*}$ is associated by $\left\langle\xi^{*} \eta, x\right\rangle=$ $(\xi \mid \eta x)$ and $a^{*} b \tau=\tau a^{*} b \in N_{*}$ is defined to be $\left(a \tau^{1 / 2}\right)^{*}\left(b \tau^{1 / 2}\right)$ for $a, b \in B$.

As a square root of this correspondence, we have a unitary map $\mathcal{H} \rightarrow L^{2}(N)$ in such a way that $|a| \tau^{1 / 2} \mapsto\left(a^{*} a \tau\right)^{1 / 2}$ for $a \in B$. Therefore, if we set $B_{+}=B \cap N_{+}$, the closure of $B_{+} \tau^{1 / 2}=\tau^{1 / 2} B_{+}$in $\mathcal{H}$ corresponds to the positive cone $L^{2}(N)_{+}$.

Related to these, we recall the following well-known and easily proved fact (cf. 7 Corollary 19.1).

Lemma 6.5. The Hilbert space $\mathcal{H}$ is canonically isomorphic to the vector space of Hilbert-Schmidt class operators with respect to $\tau$ in such a way that $\tau\left(y^{*} y\right)=$ $\left(y \tau^{1 / 2} \mid y \tau^{1 / 2}\right)$. Note that a closed operator $y$ affiliated to $N$ is in the Hilbert-Schmidt class if and only if $\tau\left(y^{*} y\right)<\infty$.

\section{Trace Formula}

We shall now utilize the Hilbert algebra structure behind $N$ to set up a method modeled after $\mathcal{N}$ to calculate the standard trace $\tau$ on $N$.

Given an open interval $I \subset[0,1 / 2]$, let $\widetilde{\mathcal{F}}_{I}$ be the set of $M$-valued analytic functions of $z \in \mathbb{R}-i I$ and set $\mathcal{F}_{I}=\cup_{\phi \in M_{*}^{+}}[\phi] \widetilde{\mathcal{F}}_{I}[\phi]$. We write $f_{\phi}(z) \phi^{i z}$ for $\phi \in M_{*}^{+}$ and $f_{\phi} \in[\phi] \mathcal{F}_{I}[\phi]$ to indicate dummies of elements in $\mathcal{F}_{I}$. All such dummies are then identified by the relation $\varphi^{i z}=\left(\varphi^{i z} \psi^{-i z}\right) \psi^{i z}$ whenever $\varphi \leq \psi$ and the obtained quotient set (which is a kind of inductive limit of dummy elements) is denoted by $\mathcal{L I}_{I}$ and an element in $\mathcal{L I}_{I}$ is called a left interpolator on $I$.

Thus each left interpolator is of the form $f(z)=f_{\varphi}(z) \varphi^{i z}$ and we say that $f(z)$ is supported by $\varphi$. Then, for $\phi \in M_{*}^{+}$mojorizing $\varphi, f(z)$ is supported by $\phi$ and $f_{\phi}(z)=f_{\varphi}(z)\left(\varphi^{i z} \phi^{-i z}\right)$, which is also denoted by $f(z) \phi^{-i z}$.

Clearly we have a similar notion of right interpolators with the obvious notations for them. These are related by the ${ }^{*}$-operation defined by $f^{*}(z)=f(-\bar{z})^{*}$ : If $f \in \mathcal{L I}_{I}, f^{*} \in \mathcal{R} \mathcal{I}_{I}$ so that $\phi^{-i z} f^{*}(z)=\left(f(-\bar{z}) \phi^{i \bar{z}}\right)^{*}$.

A pair $(l(z), r(z))$ of left and right interpolators on $I$ is called an interpolator if one can find $\phi \in M_{*}^{+}$which supports $l, r$ and interrelates them in the following sense: For each $w \in \mathbb{R}-i I$, the function $\sigma_{t}^{\phi}\left(\phi^{-i w} r(w)\right)$ of $t \in \mathbb{R}$ is analytically extended up to the horizontal line $w+\mathbb{R}$ so that the function $\sigma_{z}^{\phi}\left(\phi^{-i w} r(w)\right)$ is $\mathrm{w}^{*}$-analytic on $D=\left\{(z, w) \in \mathbb{C}^{2} ; w \in \mathbb{R}-i I, \operatorname{Im} w \leq \operatorname{Im} z \leq 0\right\}$ and satisfies $\sigma_{w}^{\phi}\left(\phi^{-i w} r(w)\right)=l(w) \phi^{-i w}$. Here, for $z \in \mathbb{C} \backslash \mathbb{R}$ and $a \in M, \sigma_{z}(a)$ means that $\sigma_{t}(a)$ $(t \in \mathbb{R})$ is analytically extended to a $\mathrm{w}^{*}$-continuous function of $\zeta \in \mathbb{R}+i \operatorname{Im} z[0,1]$ and it is evaluated at $\zeta=z$.

Since analytical extensions are moved back to the starting horizontal lines, the condition is symmetrical in the left-and-right: $\sigma_{-t}^{\phi}\left(l(w) \phi^{-i w}\right)$ is analytically extended to $\sigma_{w-z}^{\phi}\left(\phi^{-i w} r(w)\right)$, which is $\mathrm{w}^{*}$-continuous in $(z, w) \in D$. For $(z, w) \in T_{I}$, the relation $\sigma_{z+w}^{\phi}\left(\phi^{-i(z+w)} r(z+w)\right)=l(z+w) \phi^{-i(z+w)}$ is then rewritten into $\sigma_{w}^{\phi}\left(\phi^{-i(z+w)} r(z+w)\right)=\sigma_{-z}^{\phi}\left(l(z+w) \phi^{-i(z+w)}\right)$, which is a $\mathrm{w}^{*}$-analytic function of $(z, w) \in T_{I}$ and denoted by $\phi^{-i z} f(z+w) \phi^{-i w}$ when $(l(z), r(z))$ is symbolically expressed by $f(z)$. 
Moreover, the interrelating condition is compatible with the majorization changes: Let $\phi \leq \omega$ and $z \in \mathbb{R}-i I$. Then

$$
\sigma_{t}^{\omega}\left(\omega^{-i z} r(z)\right)=\left(\omega^{-i(z-t)} \phi^{i(z-t)}\right) \sigma_{t}^{\phi}\left(\phi^{-i z} r(z)\right) \phi^{i t} \omega^{-i t}
$$

is analytically continued from $t$ to $z$ to get $\left(l(z) \phi^{-i z}\right)\left(\phi^{i z} \omega^{-i z}\right)=l(z) \omega^{-i z}$.

We say that an interpolator $f(z)=(l(z), r(z))$ is supported by $\phi \in M_{*}^{+}$if both $l(z)$ and $r(z)$ are supported by $\phi$ and, in that case, we write ${ }_{\phi} f(z)=\phi^{-i z} f(z)=$ $\phi^{-i z} r(z)$ and $f_{\phi}(z)=f(z) \phi^{-i z}=l(z) \phi^{-i z}$.

Let $\mathcal{I}_{I}$ be the set of interpolators on $I$. By restriction or extension, $\mathcal{I}_{J} \subset \mathcal{I}_{I}$ if $I \subset J \subset(0,1 / 2)$. The ${ }^{*}$-operation on $\mathcal{I}_{I}$ is defined by $(l(z), r(z))^{*}=\left(r^{*}(z), l^{*}(z)\right)$ so that it is compatible with the inclusions $\mathcal{I}_{J} \subset \mathcal{I}_{I}$. Notice that $\mathcal{N}$ can be regarded as a ${ }^{*}$-subspace of $\mathcal{I}_{(0,1 / 2)}$.

Given an asymptotic function $\rho: \mathbb{R} \backslash[-R, R] \rightarrow[0, \infty)$ with $R>0$ a positive real, an interpolator $f(z)$ on $I$ is said to have a $\rho$-growth and denoted by $f(z)=$ $O(\rho(\operatorname{Re} z))$ if we can find $C>0$ so that $\left\|\phi^{-i z} f(z+w) \phi^{-i w}\right\| \leq C \rho(\operatorname{Re}(z+w))$ for any $(z, w) \in T_{I}$ satisfying $z+w \in \mathbb{R} \backslash[-R, R]-i I$. Note that the growth condition is well-defined thanks to the half-power analyticity for majorization.

An interpolator $f$ is said to be of sub-gaussian growth if, for any small $\epsilon>0$, $f(z) \phi^{-i z}=O\left(e^{\epsilon(\operatorname{Re} z)^{2}}\right)$. Let $\mathcal{I}_{I}^{g}$ be the set of interpolators of sub-gaussian growth.

For $f \in \mathcal{I}_{I}^{g}$ with $I=(\alpha, \beta) \subset[0,1 / 2]$, we here introduce a sesqui-linear form on $\mathcal{N}$ as follows. Continuous functions

$$
F(s, t)=\left(h(t-i / 2) \mid f_{\phi}(s-i r) \phi^{i t+1 / 2}{ }_{\phi} g(t-s+i r-i / 2)\right)
$$

of $(s, t) \in \mathbb{R}^{2}$ parametrized by $r \in I$ are of Gaussian decay with their absolutely convergent integrals independent of $r \in I$ owing to Cauchy's integral theorem. Moreover $F(s, t)$ does not depend on the choice of supporting $\phi$ either.

Thus a sesqui-linear form $\langle\mid\rangle_{f}$ on $\mathcal{N}$ is well-defined by

$$
\begin{aligned}
\langle h \mid g\rangle_{f} & =\int_{\mathbb{R}^{2}} d s d t\left(h(t-i / 2) \mid f_{\phi}(s-i r) \phi^{i t+1 / 2}{ }_{\phi} g(t-s+i r-i / 2)\right) \\
& =\int_{\mathbb{R}^{2}} d s d t\left(h(t-i / 2)\left(g^{*}\right)_{\phi}(-t+s+i r-i / 2) \phi^{i(s-t)} \mid f_{\phi}(s-i r) \phi^{i s+1 / 2}\right)
\end{aligned}
$$

as far as $r \in I$ and $\phi \in M_{*}^{+}$supports $f$ and $g$, which behaves well under the *-operation: $\langle g \mid h\rangle_{f^{*}}=\overline{\langle h \mid g\rangle_{f}}$. Notice that, when $f \in \mathcal{N},\langle h \mid g\rangle_{f}$ is reduced to $\left(h \tau^{1 / 2} \mid f g \tau^{1 / 2}\right)$.

We interprete the sequilinear form $\langle\mid\rangle_{f}$ as defining an operator $K$ in a kernel form by $\left(h \tau^{1 / 2} \mid K\left(g \tau^{1 / 2}\right)\right)=\langle h \mid g\rangle_{f}$, which is referred to as the virtual operator of $f(z)$ and denoted by $f$ itself.

Note that the *-operation on interpolators is compatible with the associated virtual operators; $\left(h \tau^{1 / 2} \mid f\left(g \tau^{1 / 2}\right)\right)=\overline{\left(g \tau^{1 / 2} \mid f^{*}\left(h \tau^{1 / 2}\right)\right)}$ for $g, h \in \mathcal{N}$, and virtual operators are affiliated to $N$ in the sense that $\left(h k^{*} \tau^{1 / 2} \mid f\left(g \tau^{1 / 2}\right)\right)=\left(h \tau^{1 / 2} \mid f\left(g k \tau^{1 / 2}\right)\right)$ for $g, h, k \in \mathcal{N}$.

Let $D(f)$ be the set of vectors $g \tau^{1 / 2} \in \mathcal{N} \tau^{1 / 2}$ which makes the conjugate-linear functional $h \tau^{1 / 2} \mapsto\left(h \tau^{1 / 2} \mid f\left(g \tau^{1 / 2}\right)\right)$ bounded. For $g \tau^{1 / 2} \in D(f)$, if the vector $\xi \in \mathcal{H}$ satisfying $\left(h \tau^{1 / 2} \mid \xi\right)$ is denoted by $f\left(g \tau^{1 / 2}\right)$, then we obtain a linear operator on $\mathcal{H}$ by $D(f) \ni g \tau^{1 / 2} \mapsto f\left(g \tau^{1 / 2}\right) \in \mathcal{H}$.

A virtual operator is said to be densely defined if $D(f)$ is dense in $\mathcal{H}$. When the sesqui-linear form $\langle\mid\rangle_{f}$ itself is bounded, $D(f)=\mathcal{N} \tau^{1 / 2}$ and the associated linear 
operator $\mathcal{N} \tau^{1 / 2} \rightarrow \mathcal{H}$ is bounded and identified with an element $y \in N$ in such a way that $\langle h \mid g\rangle_{f}=\left(h \tau^{1 / 2} \mid y\left(g \tau^{1 / 2}\right)\right)$ for $g, h \in \mathcal{N}$.

We next introduce the virtual vector as a conjugate-linear form on $\mathcal{N}^{2} \tau^{1 / 2}$.

Lemma 7.1. If $\phi \in M_{*}^{+}$supports $g, h \in \mathcal{N}$, then vector-valued functions $\left(h g^{*}\right)_{\phi}(s) \phi^{1 / 2}$ and $\phi^{1 / 2}{ }_{\phi}\left(h g^{*}\right)(s)$ of $s \in \mathbb{R}$ are analytically continued to $L^{2}(M)$-valued normcontinuous functions $\left(h g^{*}\right)_{\phi}(z) \phi^{1 / 2}$ and $\phi^{1 / 2}{ }_{\phi}\left(h g^{*}\right)(z)$ of $z \in \mathbb{R}-i[0,1]$ so that these are of Gaussian decay and, for $0 \leq r \leq 1 / 2$, satisfy

$$
\begin{aligned}
& \left(h g^{*}\right)_{\phi}(s-i(1-r)) \phi^{1 / 2}=\int_{\mathbb{R}} h(t-i / 2)\left(g^{*}\right)_{\phi}(-t+s+i r-i / 2) \phi^{-i t} d t, \\
& \phi^{1 / 2}{ }_{\phi}\left(h g^{*}\right)(s-i(1-r))=\int_{\mathbb{R}} \phi^{i t}{ }_{\phi} h(t+s+i r-i / 2) g^{*}(-t-i / 2) d t
\end{aligned}
$$

respectively.

Proof. We already know that $\left(h g^{*}\right)_{\phi}(s)$ has an $\mathrm{s}^{*}$-continuous analytic extension $\left(h g^{*}\right)_{\phi}(z) \in M$ to $z \in \mathbb{R}-i[0,1 / 2]$ so that $\left(h g^{*}\right)_{\phi}(s-i / 2) \phi^{1 / 2}=f(s-i / 2) \phi^{-i s}$, whereas

$$
\left(h g^{*}\right)_{\phi}(s-i / 2) \phi^{1 / 2}=\int h(t-i / 2) g_{\phi}^{*}(-t+s) \phi^{-i t} d t
$$

is analytically continued to the norm-continuous function

$$
\int h(t-i / 2) g_{\phi}^{*}(-t+z) \phi^{-i t} d t
$$

of $z \in \mathbb{R}-i[0,1 / 2]$, which is of Gaussian decay as a convolution of functions of Gaussian decay.

The sesqui-linear form $\langle h \mid g\rangle_{f}$ is now expressed by

$$
\langle h \mid g\rangle_{f}=\int_{\mathbb{R}} d s\left(\left(h g^{*}\right)_{\phi}(s-i(1-r)) \phi^{i s+1 / 2} \mid f_{\phi}(s-i r) \phi^{i s+1 / 2}\right),
$$

whenever $0<r<1$ and $\phi$ supports $g$, $h$ as well as $f$, which reveals that a conjugatelinear form $f \tau^{1 / 2}$ on $\mathcal{N}^{2} \tau^{1 / 2}$ is well-defined by the relation

$$
\left(h g^{*} \tau^{1 / 2} \mid f \tau^{1 / 2}\right)=\langle h \mid g\rangle_{f}
$$

and called the vitual vector of $f$.

Note that the virtual vector of $f^{*}$ is given by $\left(f \tau^{1 / 2}\right)^{*}$ which is defined by $\left(\xi \mid\left(f \tau^{1 / 2}\right)^{*}\right)=\overline{\left(\xi \mid f \tau^{1 / 2}\right)}$ for $\xi \in \mathcal{N}^{2} \tau^{1 / 2}$ :

$$
\langle h \mid g\rangle_{f^{*}}=\overline{\langle g \mid h\rangle_{f}}=\overline{\left(g h^{*} \tau^{1 / 2} \mid f \tau^{1 / 2}\right)}=\left(h g^{*} \tau^{1 / 2} \mid\left(f \tau^{1 / 2}\right)^{*}\right) .
$$

These are also referred to as a boundary operator and a boundary vector for $I=(0, \nu)$ and $I=(\nu, 1 / 2)$ with additional notations $\int f(t) d t$ and $\oint f(t-i / 2) d t$ respectively. We now focus on these.

Boundary Operator: In extracting linear operators from the kernel form of boundary operators, the following illustrates the meaning of boundary (limit).

Let $D$ be the set of $\mathrm{s}^{*}$-continuous sections of $\{M(i t+1 / 2)\}$ of Gaussian decay, which is a topological vector space of inductive limit of Banach spaces $D_{\delta}=$ $\left\{\{\xi(t)\} \in\{M(i t+1 / 2)\} ;\|\xi\|_{\delta}<\infty\right\}$ with $\|\xi\|_{\delta}=\sup \left\{e^{\delta t^{2}}\|\xi(t)\| ; t \in \mathbb{R}\right\}$. The 
embedding $D_{\delta} \rightarrow \mathcal{H}$ is norm-continuous and therefore so is $D \rightarrow \mathcal{H}$. For $f \in \mathcal{I}_{I}^{g}$ with $I=(0, \nu)$ and $\xi \in D_{\delta}$

$$
\int_{\mathbb{R}} f_{\varphi}(s-i r) \varphi^{i t} \xi d s
$$

is norm-convergent in $D_{\delta^{\prime}}$ for any $\delta^{\prime}<\delta$ and gives a bounded linear map $D_{\delta} \rightarrow D_{\delta^{\prime}}$, which depends continuously on $r \in I$ in the norm-topology of $\mathcal{B}\left(D_{\delta}, D_{\delta^{\prime}}\right)$. The induced continuous linear operator on $D$ is then denoted by $\int_{\mathbb{R}} f_{\varphi}(s-i r) \varphi^{i s} d s$. We say that $\int_{\mathbb{R}} f_{\varphi}(s-i r) \varphi^{i s} d s$ is bounded if it is bounded as a densely defined linear operator on $\mathcal{H}$.

Note that, if $\int_{\mathbb{R}} f_{\varphi}(s-i r) \varphi^{i s} d s \in \mathcal{B}(\mathcal{H})$ is locally norm-bounded for $r \in I$, it is s-continuous in $r \in I$ by the density of $D$ in $\mathcal{H}$.

Lemma 7.2. Let $f \in \mathcal{I}_{I}^{g}$ be supported by $\varphi \in M_{*}^{+}$. Assume that

$$
D \ni \xi \mapsto \int_{\mathbb{R}} f_{\varphi}(s-i r) \varphi^{i s} \xi d s \in \mathcal{H}
$$

gives rise to a bounded linear operator $y_{r}=\int_{\mathbb{R}} f_{\varphi}(s-i r) \varphi^{i s}$ on $\mathcal{H}$ and

$$
y=\int_{\mathbb{R}} f(s-i 0) d s=\lim _{r \rightarrow+0} \int_{\mathbb{R}} f_{\varphi}(s-i r) \varphi^{i s} d s
$$

exists in the $\mathrm{w}^{*}$-topology of $N$.

Then the boundary operator of $f(z)$ is bounded and given by the above limit.

Proof. Given $g \in \mathcal{N}$ and $\varphi \in M_{*}^{+}$, choose $\phi \in M_{*}^{+}$so that it supports $g$ and majorizes $\varphi$. Then,

$$
\mathbb{R}^{2} \ni(s, t) \mapsto \varphi^{i s} f(t-s-i / 2) \phi^{-i t} \in L^{2}(M)
$$

is analytically extended to an $L^{2}(M)$-valued norm-continuous function $\left(\varphi^{i z} \phi^{-i z}\right)\left(\phi^{i z} g(t-\right.$ $z-i / 2))$ of $z \in \mathbb{R}-i[0,1 / 2]$ and $t \in \mathbb{R}$, which is denoted by $\varphi^{i z} g(t-z-i / 2)$.

Since $\varphi^{i z} g(t-z-i / 2)=\left(\varphi^{i z} \phi^{-i z}\right) \phi^{i t+1 / 2}{ }_{\phi} g(t-z-i / 2),\left(\varphi^{i z} g\right)(t-i / 2)=$ $\varphi^{i z} g(t-z-i / 2)$ belongs to $D_{\delta}$ as a function of $t \in \mathbb{R}$ if $|g|_{\phi}(t)=O\left(e^{-\delta t^{2}}\right)$. Thus, $\xi_{r}(t)=\varphi^{r} g(t+i r-i / 2)$ is a $D_{\delta}$-valued norm-analytic function of $r$.

By our assumptions, $\mathrm{s}^{*}$-continuous family $\left\{y_{r}\right\}_{r \in I}$ in $N$ converges to $y$ in $\mathrm{w}^{*}$ topology as $r \rightarrow+0$, whence the operator norm $\left\|y_{r}\right\|$ is bounded in a neighborhood of $r=0$ and we see that

$$
y_{r} \xi_{r}=\lim _{r \rightarrow 0} y_{r} \xi_{r}=\lim _{\left(r^{\prime}, r^{\prime \prime}\right) \rightarrow(0,0)} y_{r^{\prime}} \xi_{r^{\prime \prime}}=\lim _{r^{\prime} \rightarrow 0} y_{r^{\prime}} \xi_{0}=y \xi_{0} .
$$

Now the identity

$$
\int f_{\phi}(s-i r) \phi_{\phi}^{i t+1 / 2} g(t-s+i r-i / 2) d s=\left(y_{r} \xi_{r}\right)(t)
$$

is used to get

$$
\langle h \mid g\rangle_{f}=\left(h \tau^{1 / 2} \mid y_{r} \xi_{r}\right)=\left(h \tau^{1 / 2} \mid y \xi_{0}\right)=\left(h \tau^{1 / 2} \mid y\left(g \tau^{1 / 2}\right)\right) .
$$

Corollary 7.3. Let $f(z)$ be an interpolator on $I=(0, \nu)(0<\nu \leq 1 / 2)$ and suppose that $f$ is supported by a $\phi \in M_{*}^{+}$so that $f_{\phi}(z)=f(z) \phi^{-i z}$ is a scalar operator of polynomial growth with its horizontal Fourier transform $\int_{\mathbb{R}} f_{\phi}(s-$ 
$i r) e^{i s \lambda} d s$ being in $L^{\infty}(\mathbb{R})$ for a small $r>0$ and $\mathrm{w}^{*}$-converging to $\widehat{f_{\phi}} \in L^{\infty}(\mathbb{R})$ as $r \rightarrow 0$, then the boundary operator of $f(z)$ is a bounded operator

$$
\widehat{f_{\phi}}(\log \phi)=\int_{\mathbb{R}} \widehat{f_{\phi}}(\lambda) E(d \lambda) \in N .
$$

Here $E(\cdot)$ denotes the spectral measure of $\phi^{i t}: \phi^{i t}=\int_{\mathbb{R}} e^{i t \lambda} E(d \lambda)$.

Proof. Due to the left trivialization $[\phi] L^{2}(N) \cong L^{2}(\mathbb{R}) \otimes[\phi] L^{2}(M)$, the whole thing is reduced to $L^{\infty}(\mathbb{R})$ on $L^{2}(\mathbb{R})$ and the classical harmonic analysis on the real line works.

Example 7.4. If $f(z) \phi^{-i z}$ extends to a bounded w*-continuous $M$-valued function of $z \in \mathbb{R}-i[0, \nu)$ in such a way that there exists an integrable function $\rho(t)$ satisfying $\left\|f(t-i r) \phi^{-i(t-i r)}\right\| \leq \rho(t)$ for $t \in \mathbb{R}$ and $0 \leq r<\nu$, then the boundary operator is bounded and hence belongs to $N$.

Example 7.5. For $\phi \in M_{*}^{+}$and $\mu \in \mathbb{C}$, consider an interpolator $f(z)=\frac{1}{\mu+i z} \phi^{i z}$ on $I$ with $I$ specified according to $\mu$ as follows:

(i) $I=(0,1 / 2)(\operatorname{Re} \mu \geq 0)$. The boundary operator is given by $2 \pi(1 \vee \phi)^{-\mu}$.

(ii) Either $I=(0,-\operatorname{Re} \mu)(-1 / 2<\operatorname{Re} \mu<0)$ or $I=(0,1 / 2)(\operatorname{Re} \leq-1 / 2)$.

Then the boundary operator is given by $-2 \pi(1 \wedge \phi)^{-\mu}$ for $\operatorname{Re} \mu<0$.

Here, with the help of a spectral decomposition $\phi^{i t}=\int_{\mathbb{R}} e^{i t \lambda} E(d \lambda)$,

$$
(1 \vee \phi)^{-\mu}=\int_{0}^{\infty} e^{-\mu \lambda} E(d \lambda), \quad(1 \wedge \phi)^{-\mu}=\int_{-\infty}^{0} e^{-\mu \lambda} E(d \lambda) .
$$

Boundary Vector: We next look into boundary vectors. Let $f(z) \in \mathcal{J}_{I}^{g}$ with $I=(\nu, 1 / 2)$ and $g, h \in \mathcal{N}$. In the expression

$$
\left(h g^{*} \tau^{1 / 2} \mid f \tau^{1 / 2}\right)=\int_{\mathbb{R}} d s\left(\left(h g^{*}\right)_{\varphi}(s-i(1-r)) \varphi^{i s+1 / 2} \mid f_{\varphi}(s-i r) \varphi^{i s+1 / 2}\right)
$$

( $g, h$ and $f(z)$ being supported by $\varphi \in M_{*}^{+}$), notice that the norm-convergence $\lim _{r \rightarrow 1 / 2}\left(h g^{*}\right)_{\varphi}(s-i(1-r)) \varphi^{1 / 2}=\left(h g^{*}\right)(s-i / 2) \varphi^{-i s}$ in $L^{2}(M)$ is uniformly in $s \in \mathbb{R}$ and the domination $\left\|\left(h g^{*}\right)_{\varphi}(s-i(1-r)) \varphi^{1 / 2}\right\| \leq C e^{-\delta s^{2}}$ holds uniformly in $r$, whereas $\left\|f_{\varphi}(s-i r)\right\|=O\left(e^{\epsilon s^{2}}\right)$ uniformly in $r$ for any $\epsilon>0$.

Thus, if $f(z)$ satisfies the condition that

(i) $\rho_{\varphi}(s)=\sup \left\{\left\|f_{\varphi}(s-i r)\right\| ; r \in(\nu, 1 / 2)\right\}$ is a locally integrable function of $s \in \mathbb{R}$ for some supporting $\varphi$ and

(ii) we can find a locally integrable measurable section $\eta(s) \in M(i s+1 / 2)$ so that, for a sufficiently large $\phi$ and for almost all $s, f_{\phi}(s-i r) \phi^{i s+1 / 2}$ converges weakly to $\eta(s)$ in $M(i s+1 / 2)$ as $r \rightarrow 1 / 2$,

then we have the expression

$$
\left(h g^{*} \tau^{1 / 2} \mid f \tau^{1 / 2}\right)=\int_{\mathbb{R}}\left(\left(h g^{*}\right)(s-i / 2) \mid \eta(s)\right) d s,
$$

which shows that the boundary vector of $f(z)$ is represented by the measurable section $\eta(s) \in M(i s+1 / 2)$. Note here that $\|\eta(s)\|$ is of sub-gaussian growth.

Example 7.6. If $f_{\phi}(z)$ is extended to an $M$-valued $\mathrm{w}^{*}$-continuous function of $z \in \mathbb{R}-i(\nu, 1 / 2]$, then $\rho_{\phi}(s)$ is locally bounded and $\eta(s)=f_{\phi}(s-i / 2) \phi^{i s+1 / 2}=$ $f(s-i / 2)$ meets the requirements. 
Example 7.7. Again consider $f(z)=\frac{1}{\mu+i z} \varphi^{i z}$ on $I$ but this time $I=(-\operatorname{Re} \mu, 1 / 2)$ if $-1 / 2<\operatorname{Re} \mu<0$ and $I=(0,1 / 2)$ otherwise.

Then, for $\operatorname{Re} \mu \neq-1 / 2$, the boundary vector of $f$ belongs to $\mathcal{H}$ and is given by $f(t-i / 2)=(\mu+i t+1 / 2)^{-1} \varphi^{i t+1 / 2}$.

When $\operatorname{Re} \mu \notin[-1,-1 / 2]$, the expression

$$
\left(k \tau^{1 / 2} \mid f \tau^{1 / 2}\right)=\int_{\mathbb{R}} \frac{1}{i t+\mu+1 / 2}\left\langle k^{*}(-t-i / 2) \varphi^{i t+1 / 2}\right\rangle d t
$$

for $k \in \mathcal{N}^{2}$ is analytically changed in the integration variable to get

$$
\left(k \tau^{1 / 2} \mid f \tau^{1 / 2}\right)=\int_{\mathbb{R}} \frac{\phi\left(k^{*}(-t) \varphi^{i t}\right)}{i t+\mu+1} d t .
$$

Thus the parametric limit of $f \tau^{1 / 2}$ exists in simple convergence as $\mu$ approaches to a point in $\operatorname{Re} \mu=-1 / 2$ from the right $(\operatorname{Re} \mu>-1 / 2)$.

Now let $\mu=i m-1 / 2(m \in \mathbb{R})$ be on the critical line $\operatorname{Re} \mu=-1 / 2$ and set $\epsilon=1 / 2-r$. By Lemma 7.1] we have

$$
\langle h \mid g\rangle_{f}=\int_{\mathbb{R}} \frac{1}{i(s+m)+1 / 2} \phi\left(\phi^{i s}\left(g h^{*}\right)(-s)\right) d s,
$$

which reveals that the boundary vector of $f(z)$ coincides with

$$
\lim _{\epsilon \rightarrow+0} \oint_{\mathbb{R}} \frac{1}{i(t+m)+\epsilon} \phi^{i t+1 / 2} d t .
$$

We now generalize the notion of interpolators on $I=(0,1 / 2)$ so that $f(z)$ is allowed to be not defined on a compact subset $K$ of $\mathbb{R}-i(0,1 / 2)$. The various analyticity is then defined just avoiding $K$. Since the growth condition is about horizontal asymptotics, it remains having a meaning as well.

We introduce the residue operator $R_{f}=\oint_{K} f(z) d z: \mathcal{N} \tau^{1 / 2} \rightarrow \mathcal{H}$ by

$$
R_{f}\left(g \tau^{1 / 2}\right)=\oint_{\mathbb{R}}(\oint f(z) g(t-z-i / 2) d z) d t .
$$

Here $f(z) g(t-z-i / 2)=f_{\phi}(z) \phi^{i t+1 / 2}{ }_{\phi} g(t-z-i / 2)$ is an $M(i t+1 / 2)$-valued analytic function of $z \in(\mathbb{R}-i[0,1 / 2]) \backslash K$ and the coutour integral is performed by surrounding $K$.

Theorem 7.8 (Trace Formula). Let $f(z)$ be an interpolator on $(0,1 / 2)$ of subgaussian growth and assume that the boundary vector $f \tau^{1 / 2}=\oint_{\mathbb{R}} f(t-i / 2) d t$ exists in $\mathcal{H}$.

Then the sum of the boundary operator $f$ and the residue operator $R_{f}$ is $\tau$ measurable and we have

$$
\tau\left(\left(f+R_{f}\right)^{*}\left(f+R_{f}\right)\right)=\left(f \tau^{1 / 2} \mid f \tau^{1 / 2}\right)=\int_{\mathbb{R}}(f(t-i / 2) \mid f(t-i / 2)) d t
$$

Proof. Let $V_{f}$ be the virtual operator of $f(z)(z \in \mathbb{R}-i(1 / 2-\epsilon, 1 / 2))$. By the residue formula, $V_{f}=f+R_{f}$ and, for $g, h \in \mathcal{N}$,

$$
\left(h \tau^{1 / 2} \mid V_{f}\left(g \tau^{1 / 2}\right)\right)=\left(h \tau^{1 / 2} \mid\left(f \tau^{1 / 2}\right) g\right)=\left(h^{1 / 2} \tau^{1 / 2} \mid l\left(f \tau^{1 / 2}\right)\left(g \tau^{1 / 2}\right)\right)
$$

shows that the virtual operator $V_{f}$ is closable with its closure given by $l\left(f \tau^{1 / 2}\right)$. Lemma 6.5 is then the applied to get the assertion. 
Corollary 7.9. If $f(z)$ is analytic on the whole $\mathbb{R}-i(0,1 / 2)$ additionally, then the boundary operator $f$ is $\tau$-measurable and we have

$$
\left.\tau\left(f^{*} f\right)\right)=\left(f \tau^{1 / 2} \mid f \tau^{1 / 2}\right)=\int_{\mathbb{R}}(f(t-i / 2) \mid f(t-i / 2)) d t .
$$

Example 7.10. Let $G \in L^{2}(\mathbb{R})$ and suppose that its Fourier transform $\widehat{G}(\lambda)=$ $\int_{\mathbb{R}} G(t) e^{-i \lambda t} d t$ is integrable and satisfies $\int_{0}^{\infty}|\widehat{G}(\lambda)|^{2} e^{\lambda} d \lambda<\infty$.

Then the inverse Fourier transform $G_{w}$ of $\widehat{G}(\lambda) e^{i w \lambda}$ belongs to $L^{2}(\mathbb{R}) \cap C_{0}(\mathbb{R})$ and depends on $w \in \mathbb{R}-i[0,1 / 2]$ norm-continuously for both $\|\cdot\|_{\infty}$ and $\|\cdot\|_{2}$. Since, for $F \in L^{2}(\mathbb{R})$,

$$
\left(F \mid G_{w}\right)=\frac{1}{2 \pi} \int_{\mathbb{R}} \overline{\widehat{F}(\lambda)} \widehat{G}(\lambda) e^{i w \lambda} d \lambda
$$

is analytic in $w$ and $G_{s}$ is reduced to the translation $G(t+s)$ of $G(t), F(t)$ is analytically extended to $F(z)$ so that $G_{w}(t)=G(t+w)$ for $w \in \mathbb{R}-i[0,1 / 2]$ and $t \in \mathbb{R}$.

Now, for $\phi \in M_{*}^{+}, g(z)=G(z) \phi^{i t}$ defines an interpolator on $(0,1 / 2)$ which vanishes at $\operatorname{Re} z= \pm \infty$. Since $\phi^{i t}$ on $\mathcal{H}$ is given by translation on $L^{2}(\mathbb{R}) \otimes$ $[\phi] L^{2}(M)[\phi] \cong[\phi] \mathcal{H}[\phi]$, the associated boundary operator is bounded and the boundary vector is given by $\oint_{\mathbb{R}} G(t-i / 2) \phi^{i t+1 / 2} d t$ so that

$$
\begin{aligned}
\tau\left(g^{*} f g\right) & =\int_{\mathbb{R}^{2}}(g(t-i / 2) \mid f(s) g(t-s-i / 2)) d s d t \\
& =\phi(1) \int_{\mathbb{R}^{2}} \overline{G(t-i / 2)} F(s) G(t-s-i / 2) d s d t \\
& =\frac{\phi(1)}{2 \pi} \int_{\mathbb{R}} \widehat{F}(\lambda)|\widehat{G}(\lambda)|^{2} e^{\lambda} d \lambda .
\end{aligned}
$$

Here, for $F \in L^{1}(\mathbb{R})$, an $L^{1}$-section $\{f(t)\}$ of $\{M(i t)\}$ is defined by $f(t)=F(t) \phi^{i t}$ and $f=\int_{\mathbb{R}} f(t) d t \in N$.

Thus, letting $A$ be the $\mathrm{W}^{*}$-subalgebra of $[\phi] N[\phi]$ generated by $\left\{\phi^{i t} ; t \in \mathbb{R}\right\}$, $L^{2}(A, \tau)$ is identified with $L^{2}\left(\mathbb{R}, e^{\lambda} d \lambda\right)$ by a unitary map

$$
\left.U_{\phi}: L^{2}(A, \tau) \ni g \tau^{1 / 2}\right) \mapsto \sqrt{\frac{\phi(1)}{2 \pi}} \widehat{G}(\lambda) \in L^{2}\left(\mathbb{R}, e^{\lambda} d \lambda\right)
$$

so that $\phi^{i s}$ on $L^{2}(A, \tau)$ is realized by a multiplication of the function $e^{-i s \lambda}$ of $\lambda \in \mathbb{R}$.

Example 7.11. For $-1 / 2<\operatorname{Re} \beta<0$ and $\phi \in M_{*}^{+}$, the interpolator $f(z)=$ $\frac{1}{\beta+i z} \phi^{i z}$ has $-2 \pi(1 \wedge \phi)^{-\beta} \in N$ as the boundary operator. The residue operator is calculated by the realization $L^{\infty}(A)$ on $L^{2}(A)$ as

$$
\int_{|z-i \beta|=\epsilon} \frac{1}{\beta+i z} e^{i \lambda z} d z=2 \pi e^{-\beta \lambda}
$$

which is therefore $2 \pi \phi^{-\beta}$. Adding these, we see that $(1 \vee \phi)^{-\beta}$ is in the HilbertSchmidt class and hence, for $x \in M$ and $\mu=-r+i s \in-(0,1)+i \mathbb{R}, x(1 \vee \phi)^{-\mu}=$ $x(1 \vee \phi)^{r / 2-i s}(1 \vee \phi)^{r / 2}$ is in the trace class with

$$
\begin{aligned}
2 \pi \tau\left(x(1 \vee \phi)^{-\mu}\right) & =\phi(x) \int_{\mathbb{R}} \frac{1}{-i t+(1-r) / 2} \frac{1}{i(t+s)+(1-r) / 2} d t \\
& =\frac{\phi(x)}{i s-r+1}=\frac{\phi(x)}{\mu+1} .
\end{aligned}
$$


Although Haagerup deals only with the case $\mu=0$ and its scaled variation, the following generalization should also be attributed to him.

Theorem 7.12 (Haagerup's Trace Formula). Let $\omega$ be a weight on $M$ in our sense. The trace of a positive operator $(1 \vee \omega)^{-\mu}$ with $\mu \in \mathbb{R}$, which belongs to $N$ for $\mu \geq 0$ and is affiliated to $N$ for $\mu<0$, is given by

$$
\tau\left((1 \vee \omega)^{-\mu}\right)= \begin{cases}\frac{\omega(1)}{2 \pi(\mu+1)} & \text { if } \mu>-1, \\ \infty & \text { otherwise. }\end{cases}
$$

Moreover, when $\omega \in M_{*}^{+}$, for any $x \in M$ and $\mu \in(-1, \infty)+i \mathbb{R}$, the $\tau$-measurable operator $x(1 \vee \omega)^{-\mu}$ is in the trace class and we have

$$
\tau\left(x(1 \vee \omega)^{-\mu}\right)=\frac{\omega(x)}{2 \pi(\mu+1)} .
$$

Proof. Assume $\omega \in M_{*}^{+}$. Then $\omega^{i t}$ is realized as a multiplication operator on $L^{2}\left(\mathbb{R}, e^{\lambda} d \lambda\right)$ by a function $e^{-i t \lambda}$ of $\lambda \in \mathbb{R}$. Consequently $(1 \vee \omega)^{-\mu}$ is represented by the function $1_{(-\infty, 0]}(\lambda) e^{\lambda \mu}$ of $\lambda$, which is integrable relative to the measure $e^{\lambda} d \lambda$ if and only if $\operatorname{Re} \mu>-1$ with

$$
\int_{-\infty}^{0} e^{\lambda \mu} e^{\lambda} d \lambda=\frac{1}{\mu+1}
$$

Since our weights are orthogonal sums of elements in $M_{*}^{+}$, the formula for $\omega \in$ $M_{*}^{+}$remains valid for weights.

The remaining part is already covered in Example 7.11 Remark 6.

(i) By the integral expression $\int_{\mathbb{R}} \frac{1}{\mu+i t} \omega^{i t} d t$ of $2 \pi(1 \vee \omega)^{-\mu}$, the formula coincides with the one obtained from the formal argument.

(ii) The normalization of our trace is different from that in [6] and [9] by a factor $2 \pi$.

Thus, for $\omega \in M_{*}^{+}$, the analytic generator $h$ of $\omega^{i t}$ as a positive operator on $\mathcal{H}$, which satisfies $\theta_{s}(h)=e^{-s} h$ (called relative invariance of degree -1 ), is $\tau$ measurable in the sense that $\lim _{r \rightarrow \infty} \tau([r \vee h])=0$. Haagerup's ingeneous observation is that the whole $L^{p}(M)$ 's are captured as measurable operators on $\mathcal{H}$ satisfying relative invariance of degree $-1 / p$.

We now go into the reverse problem of characterizing $\tau$-measurable positive operators satisfying relative invariance of degree -1 , which is the heart of Haagerup's correspondence.

Recall the original approach to this problem: First establish a one-to-one correspondence between normal weights on $M$ and $\theta$-invariant normal weights on $N$. Second the latter is then paraphrased into positive operators of relative invariance of degree -1 by taking Radon-Nikodym derivative with respect to $\tau$. Finally, positive operators associated to $M_{*}^{+}$are characterised as $\tau$-measurable operators among these.

Formally the whole processes look natural and seem harmless but it is in fact supported by clever and effective controls over infinities based on extended positive parts.

We shall here present an inelegant but down-to-earth proof by continuing elementary Fourier calculus. 


\section{HaAgerup Correspondence}

Let $h \geq 0$ be a $\tau$-measurable operator on $\mathcal{H}$ satisfying $\theta_{s}(h)=e^{-s} h$ for $s \in \mathbb{R}$. Our first task here is to identify $h^{i t}$ with $\varphi^{i t}$ for some $\varphi \in M_{*}^{+}$.

Let $e=[1 \vee h]$ be the support projection of $1 \vee h$. By the relative invariance of $h, \theta_{s}(e)$ is the support projection of $e^{s} \vee h$ and we have a Stieltjes integral representation of $h$

$$
h=-\int_{-\infty}^{\infty} e^{s} d \theta_{s}(e)=\int_{-\infty}^{\infty} e^{-s} d \theta_{-s}(e)
$$

and set

$$
(1 \vee h)^{-\mu}=-\int_{0}^{\infty} e^{-\mu s} d \theta_{s}(e),
$$

which is $\tau$-measurable for any $\mu \in \mathbb{C}$ in view of $\tau(e)<\infty$. Notice that $\theta_{s}(e)$ is continuous in $s \in \mathbb{R}$ and $d \theta_{s}$ has no spectral jumps.

Let $x \in M$ and start with the computation

$$
\begin{aligned}
\tau\left(h x(1 \vee h)^{-\mu}\right) & =\tau\left(x(1 \vee h)^{-\mu} h\right)=\tau\left(x(1 \vee h)^{1-\mu}\right) \\
& =-\int_{0}^{\infty} e^{(1-\mu) s} d \tau\left(x \theta_{s}(e)\right)=-\int_{0}^{\infty} e^{(1-\mu) s} d\left(e^{-s}\right) \tau(x e) \\
& =\tau(x e) \int_{0}^{\infty} e^{-\mu s} d s=\frac{1}{\mu} \tau(x e),
\end{aligned}
$$

which is valid for $\operatorname{Re} \mu>0$.

For $t \in \mathbb{R}, \sigma_{t}(x)=h^{i t} x h^{-i t}(x \in M)$ defines an automorphic action of $\mathbb{R}$ on $M$ because $h^{i t} x h^{-i t}$ is $\theta$-invariant in view of $\theta_{s}\left(h^{i t}\right)=e^{-i s t} h^{i t}$. We claim that $\varphi(x)=2 \pi \tau(x e)$ satisfies the KMS-condition for the automorphic action $\sigma_{t}$.

First notice that $[h]=[\varphi]$. In fact, from the definition of $\varphi$ and the faithfulness of the standard trace, $(1-[\varphi]) e=0$, which means that $e \leq[\varphi]$ and then $[h]=$ $\lim _{s \rightarrow-\infty} \theta_{s}(e) \leq \theta_{s}([\varphi])=[\varphi]$. Conversely, from $(1-[h]) e=0,1-[h] \leq 1-[\varphi]$ gives the reverse inequality.

Now consider $\varphi\left(x^{*} \sigma_{t}(x)\right)=\tau\left(x^{*} h^{i t} x h^{-i t} e\right)$ with $x \in M$. If the Stieltjes integral expression for $h$ is used as in $x h^{-i t} e=-\int_{0}^{\infty} e^{-i s t} d \theta_{s}(x e)$, we have

$$
\begin{aligned}
-\frac{1}{2 \pi} \varphi\left(x^{*} \sigma_{t}(x)\right) & =\int_{0}^{\infty} e^{-i s t} d \tau\left(x^{*} h^{i t} \theta_{s}(x e)\right) \\
& =\int_{0}^{\infty} e^{-i s t} d \tau\left(\theta_{s}\left(x^{*} \theta_{-s}\left(h^{i t}\right) x e\right)\right) \\
& =\int_{0}^{\infty} e^{-i s t} d\left(e^{-s} e^{i s t}\right) \tau\left(x^{*} h^{i t} x e\right)=(i t-1) \tau\left({ }^{*} h^{i t} x e\right)
\end{aligned}
$$

and then

$$
-\tau\left(x^{*} h^{i t} x e\right)=\int_{-\infty}^{0} e^{i s t} d \tau\left(x^{*} \theta_{s}(e) x e\right)+\int_{0}^{\infty} e^{i s t} d \tau\left(x^{*} \theta_{s}(e) x e\right),
$$

together with

$$
\begin{aligned}
& \int_{0}^{\infty} e^{i s t} d \tau\left(x^{*} \theta_{s}(e) x e\right)=\int_{0}^{\infty} e^{i s t} d\left(e^{-s} \tau\left(x^{*} e x \theta_{-s}(e)\right)\right) \\
= & \int_{0}^{\infty} e^{i s t} e^{-s} d \tau\left(x^{*} e x \theta_{-s}(e)\right)-\int_{0}^{\infty} e^{i s t} e^{-s} \tau\left(x^{*} e x \theta_{-s}(e)\right) d s
\end{aligned}
$$


reveals that $-\tau\left(x^{*} h^{i t} x e\right)$ is analytically extended to a bounded continuous function

$$
\begin{gathered}
-\tau\left(x^{*} h^{i z} x e\right)=\int_{-\infty}^{\infty} e^{i s z} d \tau\left(x^{*} \theta_{s}(e) x e\right)=\int_{-\infty}^{\infty} e^{i s z} d\left(e^{-s} \tau\left(x^{*} e x \theta_{-s}(e)\right)\right) \\
=\int_{-\infty}^{\infty} e^{i s z} e^{-s} d \tau\left(x^{*} e x \theta_{-s}(e)\right)-\int_{-\infty}^{\infty} e^{i s z} e^{-s} \tau\left(x^{*} e x \theta_{-s}(e)\right) d s .
\end{gathered}
$$

of $z=t-i r \in \mathbb{R}-i[0,1]$.

In these and the following calculations, note that $\tau\left(x^{*} e x \theta_{-s}(e)\right)\left(\tau\left(x^{*} \theta_{s}(e) x e\right)\right)$ is positive, increasing (decreasing) and continuous in $s \in \mathbb{R}$, whence both $d \tau\left(x^{*} e x \theta_{-s}(e)\right)$ and $-d \tau\left(x^{*} \theta_{s}(e) x e\right)$ give rise to positive finite measures on $\mathbb{R}$.

Consequently, with the notation $\varphi\left(x^{*} \sigma_{z}(x)\right)$ for the analytic continuation of $\varphi\left(x^{*} \sigma_{t}(x)\right)$ and, with the help of integration-by-parts, we get the expression

$$
\begin{aligned}
& \frac{1}{2 \pi} \varphi\left(x^{*} \sigma_{t-i r}(x)\right)=(i t+r-1) \int_{-\infty}^{\infty} e^{(i t+r-1) s} d \tau\left(x^{*} e x \theta_{-s}(e)\right) \\
& -(i t+r-1) \int_{-\infty}^{\infty} e^{(i t+r-1) s} \tau\left(x^{*} e x \theta_{-s}(e)\right) d s \\
& =(i t+r) \int_{-\infty}^{\infty} e^{(i t+r-1) s} d \tau\left(x^{*} \operatorname{ex} \theta_{-s}(e)\right)-\left[e^{(i t+r-1) s} \tau\left(x^{*} e x \theta_{-s}(e)\right)\right]_{-\infty}^{\infty} .
\end{aligned}
$$

For $0<r<1$, we see $\lim _{s \rightarrow \infty} e^{(i t+r-1) s} \tau\left(x^{*} \operatorname{ex} \theta_{-s}(e)\right)=0$ and

$$
\lim _{s \rightarrow-\infty} e^{(i t+r-1) s} \tau\left(x^{*} e x \theta_{-s}(e)\right)=\lim _{s \rightarrow-\infty} e^{(i t+r) s} \tau\left(x^{*} \theta_{s}(e) x e\right)=0
$$

at the boundary values and therefore

$$
\frac{1}{2 \pi} \varphi\left(x^{*} \sigma_{t-i r}(x)\right)=(i t+r) \int_{-\infty}^{\infty} e^{(i t+r-1) s} d \tau\left(x^{*} e x \theta_{-s}(e)\right) .
$$

Since both sides are continuous in $r \in[0,1]$, the equality holds at the boundary as well. We now compare this expression with

$$
\begin{aligned}
\frac{1}{2 \pi} \varphi\left(\sigma_{t}(x) x^{*}\right) & =\tau\left(e h^{i t} x h^{-i t} x^{*}\right)=-\int_{0}^{\infty} e^{i s t} d \tau\left(\theta_{s}(e) x h^{-i t} x^{*}\right) \\
& =-\int_{0}^{\infty} e^{i s t} d \tau\left(\theta_{s}\left(e x \theta_{-s}\left(h^{-i t}\right) x^{*}\right)\right) \\
& =-\int_{0}^{\infty} e^{i s t} d\left(e^{-s-i s t}\right) \tau\left(e x h^{-i t} x^{*}\right)=(i t+1) \tau\left(e x h^{-i t} x^{*}\right) \\
& =(i t+1) \int_{-\infty}^{\infty} e^{i s t} d \tau\left(e x \theta_{-s}(e) x^{*}\right)
\end{aligned}
$$

to conclude that $\varphi\left(x^{*} \sigma_{t-i}(x)\right)=\varphi\left(\sigma_{t}(x) x^{*}\right)$ for $t \in \mathbb{R}$.

So far we have checked that $h^{i t} x h^{-i t}=\varphi^{i t} x \varphi^{-i t}$ for $x \in[\varphi] M[\varphi]$. Then $u(t)=$ $h^{i t} \varphi^{-i t}$ is a unitary in the center of $[\varphi] M[\varphi]$. Since each $\varphi^{i t}$ commutes with the reduced center, $\{u(t)\}$ is a one-parameter group of unitaries in the reduced algebra. Let $u(t)=\int_{\mathbb{R}} e^{i s t} E(d s)$ be the spectral decomposition in $[\varphi] M[\varphi]$. Then $a_{n}=$ $\int_{[-n, n]} e^{s / 2} E(d s)$ is an increasing sequence of positive elements in the reduced center and $\varphi_{n}=a_{n} \varphi a_{n} \in M_{*}^{+}$satisfies $\varphi_{n}^{i t}=h^{i t}\left[a_{n}\right]=\left[a_{n}\right] h^{i t}$ for $t \in \mathbb{R}$. Set $h_{n}=h\left[a_{n}\right]=$ 
$\left[a_{n}\right] h$, which is also $\tau$-measurable and satisfies $\theta_{s}\left(h_{n}\right)=e^{-s} h_{n}$. From the equalities

$$
\frac{\varphi_{n}(x)}{2 \pi \mu}=\tau\left(x\left(1 \vee \varphi_{n}\right)^{1-\mu}\right)=\tau\left(x\left(1 \vee h_{n}\right)^{1-\mu}\right)=\tau\left(x\left[a_{n}\right](1 \vee h)^{1-\mu}\right)=\frac{\varphi\left(x\left[a_{n}\right]\right)}{2 \pi \mu}
$$

for $x \in M$ and $\mu \geq 1$, one sees that $\varphi_{n}=\varphi\left[a_{n}\right]=\left[a_{n}\right] \varphi$ and then $\varphi_{n}^{i t}=\varphi^{i t}\left[a_{n}\right]$ for $t \in \mathbb{R}$. Finally we have $h^{i t}=\lim _{n \rightarrow \infty} h_{n}^{i t}=\lim _{n \rightarrow \infty} \varphi^{i t}\left[a_{n}\right]=\varphi^{i t}$.

We next check the additivity of the correspondence $h_{\varphi} \leftrightarrow \varphi$. To see this, we first establish the following relation.

Lemma 8.1. Let $\omega \in M_{*}^{+}$and $\mu>0$. Then

$$
(1 \vee \omega)^{-\mu}=\frac{1}{2 \pi} \int_{\mathbb{R}} \frac{1}{\mu+i t} \omega^{i t} d t
$$

is in the $\tau$-trace class and, for $x \in[\varphi] M$, we have

$$
\tau\left(h x^{*}(1 \vee \omega)^{-\mu} x\right)=\frac{1}{2 \pi \mu} \varphi\left(x^{*} x\right) .
$$

Recall here that $(1 \vee \omega)^{-\mu / 2}=\frac{1}{2 \pi} \int_{\mathbb{R}} \frac{1}{i t+\mu / 2} \omega^{i t} d t$ belongs to $B_{+}$in such a way that

$$
(1 \vee \omega)^{-\mu / 2} \tau^{1 / 2}=\frac{1}{2 \pi} \oint_{\mathbb{R}} \frac{1}{i t+(\mu+1) / 2} \omega^{i t+1 / 2} d t
$$

The identity is checked as follows: Letting $y=x^{*}(1 \vee \omega)^{-\mu} x$, we have

$$
\begin{aligned}
\tau(h y) & =-\lim _{n \rightarrow \infty} \int_{-n}^{n} e^{s} d \tau\left(\theta_{s}(e) y\right) \\
& =\lim _{n \rightarrow \infty}\left(\int_{-n}^{n} e^{s} \tau\left(\theta_{s}(e) y\right) d s-e^{n} \tau\left(\theta_{n}(e) y\right)+e^{-n} \tau\left(\theta_{-n}(e) y\right)\right) \\
& =\lim _{n \rightarrow \infty}\left(\int_{-n}^{n} d s e^{s} \int_{\mathbb{R}} d t \frac{1}{2 \pi(\mu+i t)} \tau\left(\theta_{s}(e) x^{*} \omega^{i t} x\right)-\tau\left(e \theta_{-n}(y)\right)\right) \\
& =\lim _{n \rightarrow \infty}\left(\int_{-n}^{n} d s \int_{\mathbb{R}} d t \frac{e^{i s t}}{2 \pi(\mu+i t)} \tau\left(e x^{*} \omega^{i t} x\right)-\frac{1}{2 \pi} \int_{\mathbb{R}} \frac{e^{i n t}}{\mu+i t} \tau\left(e x^{*} \omega^{i t} x\right) d t\right) .
\end{aligned}
$$

By the lemma below, the function $\tau\left(e x^{*} \omega^{i t} x\right) /(\mu+i t)$ is integrable, whence

$$
\lim _{n \rightarrow \infty} \int_{\mathbb{R}} \frac{e^{i n t}}{\mu+i t} \tau\left(e x^{*} \omega^{i t} x\right) d t=0
$$

Lemma 8.2. We have

$$
\tau\left(e x^{*} \omega^{i t} x\right)=\frac{1}{2 \pi(1-i t)} \varphi\left(x^{*} \omega^{i t} x \varphi^{-i t}\right) .
$$

Proof. From the expression $\tau\left(e x^{*} \omega^{i s} x\right)=\left(x e \tau^{1 / 2} \mid \omega^{i s} x e \tau^{1 / 2}\right)$ with

$$
\begin{aligned}
& \omega^{i s} x e \tau^{1 / 2}=\frac{1}{2 \pi} \oint_{\mathbb{R}} d t \frac{1}{i(t-s)+1 / 2} \omega^{i s} x \varphi^{-i s} \varphi^{i t+1 / 2}, \\
\tau\left(e x^{*} \omega^{i s} x\right)= & \frac{1}{(2 \pi)^{2}} \int_{\mathbb{R}} d t \frac{1}{-i t+1 / 2} \frac{1}{i(t-s)+1 / 2}\left(x \varphi^{i t+1 / 2} \mid \omega^{i s} x \varphi^{-i s} \varphi^{i t+1 / 2}\right) \\
= & \frac{1}{(2 \pi)^{2}} \int_{\mathbb{R}} d t \frac{1}{-i t+1 / 2} \frac{1}{i(t-s)+1 / 2} \varphi\left(x^{*} \omega^{i s} x \varphi^{-i s}\right) \\
& =\frac{1}{2 \pi} \frac{1}{1-i s} \varphi\left(x^{*} \omega^{i s} x \varphi^{-i s}\right),
\end{aligned}
$$


To deal with the first term in the last expression of $\tau(h y)$, we use the relation $2 \pi(\mu+i t)^{-1}=g^{*} * g$ for $g(t)=1 /(i t+\mu / 2)$ to see that

$$
\int_{\mathbb{R}} \frac{e^{i s t}}{\mu+i t} \omega^{i t} d t=\int_{\mathbb{R}} d t^{\prime} e^{-i s t^{\prime}} \overline{g\left(t^{\prime}\right)} \omega^{-i t^{\prime}} \int_{\mathbb{R}} d t e^{i s t} g(t) \omega^{i t}
$$

and hence

$$
\begin{aligned}
2 \pi \int_{\mathbb{R}} \frac{e^{i s t}}{\mu+i t} & \left(x \xi_{0} \mid \omega^{i t} x \xi_{0}\right) d t \\
& =\left(\int_{\mathbb{R}} d t^{\prime} e^{i s t^{\prime}} g\left(t^{\prime}\right) \omega^{i t^{\prime}} x \xi_{0} \mid \int_{\mathbb{R}} d t e^{i s t} g(t) \omega^{i t} x \xi_{0}\right) \\
& =\sum_{j}\left(\int_{\mathbb{R}} d t^{\prime} e^{i s t^{\prime}} g\left(t^{\prime}\right) \omega^{i t^{\prime}} x \xi_{0} \mid \delta_{j}\right)\left(\delta_{j} \mid \int_{\mathbb{R}} d t e^{i s t} g(t) \omega^{i t} x \xi_{0}\right) \\
& =\sum_{j} \int_{\mathbb{R}} d t e^{i s t}\left(F_{j}^{*} * F_{j}\right)(t)=\sum_{j}\left|\widehat{F}_{j}(s)\right|^{2},
\end{aligned}
$$

where $\left\{\delta_{j}\right\}$ is an orthonormal system in $\mathcal{H}$ supporting vectors $\left\{\omega^{i t} x \xi_{0}\right\}_{t \in \mathbb{R}}$ and $F_{j}(t)=g(t)\left(\delta_{j} \mid \omega^{i t} x \xi_{0}\right)$ together with their Fourier transforms $\widehat{F}_{j}(s)=\int_{\mathbb{R}} e^{i s t} F_{j}(t) d t$ belong to $L^{2}(\mathbb{R})$.

The Plancherel formula is then applied to each $F_{j}$ to get

$$
\begin{aligned}
(2 \pi)^{2} \tau(h y) & =\int_{-\infty}^{\infty} \sum_{j}\left|\widehat{F_{j}}(s)\right|^{2} d s=\sum_{j} \int_{-\infty}^{\infty}\left|\widehat{F_{j}}(s)\right|^{2} d s=2 \pi \sum_{j} \int_{\mathbb{R}}\left|F_{j}(t)\right|^{2} d t \\
& =2 \pi \int_{\mathbb{R}} \sum_{j}\left|F_{j}(t)\right|^{2} d t=\frac{2 \pi}{\mu}\left(x \xi_{0} \mid x \xi_{0}\right)=\frac{2 \pi}{\mu} \tau\left(e x^{*} x\right) .
\end{aligned}
$$

Similarly and more easily, the side identity follows from

$$
\begin{aligned}
2 \pi \int_{\mathbb{R}}\left(\xi \mid \theta_{s}(y) \xi\right) & =\int_{\mathbb{R}} d s \int_{\mathbb{R}} d t \frac{1}{\mu+i t}\left(x \xi \mid \theta_{s}\left(\omega^{i t}\right) x \xi\right) \\
& =\int_{\mathbb{R}} d s \int_{\mathbb{R}} d t \frac{e^{-i s t}}{\mu+i t}\left(x \xi \mid \omega^{i t} x \xi\right)=\frac{2 \pi}{\mu}\left(\xi \mid x^{*} x \xi\right)
\end{aligned}
$$

for each $\xi \in L^{2}(N)$.

Theorem 8.3 (Haagerup correspondence). There is a linear isomorphism between $M_{*}$ and the linear space of $\tau$-measurable operators $h$ on $L^{2}(N)$ satisfying $\theta_{s}(h)=$ $e^{-s} h$ and so that $\varphi \in M_{*}^{+}$corresponds to the analytic generator $h_{\varphi}$ of the oneparameter group $\left\{\varphi^{i t}\right\}$ of partial isometries in $N$.

Moreover the correspondence preserves $N^{*}$-bimodule structures as well as positivity.

Proof. The correspondence is already established for positive parts and Lemma 8.1 is used to get the additivity by

$$
\frac{1}{2 \pi \mu} \phi\left(x^{*} x\right)=\left\langle\left(h_{\varphi}+h_{\psi}\right) x^{*}(1 \vee \omega)^{-\mu} x\right\rangle=\frac{1}{2 \pi \mu}\left(\varphi\left(x^{*} x\right)+\psi\left(x^{*} x\right)\right) .
$$

Here $\varphi, \psi \in M_{*}^{+}$and $\phi \in M_{*}^{+}$is specified by $h_{\phi}=h_{\varphi}+h_{\psi}$.

Once the semilinearity is obtained, the other part is almost automatic. The linear extension is well-defined by $h_{\varphi}=h_{\varphi_{1}}-h_{\varphi_{2}}+i h_{\varphi_{3}}-i h_{\varphi_{4}}$ for $\varphi=\varphi_{1}-\varphi_{2}+i \varphi_{3}-i \varphi_{4} \in$ 
$M_{*}$ with $\varphi_{j} \in M_{*}^{+}$. The identity $a h_{\varphi} a^{*}=h_{a \varphi a^{*}}$ for $a \in M$ follows again from Lemma 8.1 as

$$
\tau\left(a h a^{*} x^{*}(1 \vee \omega)^{-\mu} x\right)=\tau\left(h a^{*} x^{*}(1 \vee \omega)^{-\mu} x a\right)=\frac{2 \pi}{\mu} \varphi\left(a^{*} x^{*} x a\right)=\frac{2 \pi}{\mu}\left(a \varphi a^{*}\right)\left(x^{*} x\right)
$$

and then $a h_{\varphi} b^{*}=h_{a \varphi b^{*}}$ by polarization.

\section{REFERENCES}

[1] O. Bratteli and D.W. Robinson, Operator algebras and quantum statistical mechanics, Vol.1, Springer, 1979

[2] U. Haagerup, On the dual weights for crossed products of von Neumann algebras I, Math. Scand., 43(1978), 99-118.

[3] U. Haagerup, On the dual weights for crossed products of von Neumann algebras II, Math. Scand., 43(1978), 119-140.

[4] U. Haagerup, Operator valued weights in von Neumann algebras I, J. Funct. Anal., 32(1979), $175-206$.

[5] U. Haagerup, Operator valued weights in von Neumann algebras II, J. Funct. Anal., 33(1979), 339-361.

[6] U. Haagerup, $L^{p}$-spaces associated with an arbitrary von Neumann algebra, Colloques Internationaux CNRS, No. 274, 175-184, 1979.

[7] I.E. Segal, A non-commutative extension of abstract integration, Annal Math., 57(1953), 401-457.

[8] M. Takesaki, Theory of operator algebras, Vol.2, Springer, 2003.

[9] M. Terp, $L^{p}$-spaces associated with von Neumann algebras, Kbenhavns Univ., 1981.

[10] S. Yamagami, Algebraic aspects in modular theory, Publ. RIMS, 28(1992), 1075-1106.

[11] S. Yamagami, Modular theory for bimodules, J. Funct. Anal., 125(1994), 327-357. 